\title{
Valeurs spéciales de paramètres de diagrammes de Diamond
}

\author{
Yongquan $\mathrm{HU}$
}

Résumé. - Soit $L$ une extension finie non ramifiée de $\mathbb{Q}_{p}$ et $\bar{\rho}: \operatorname{Gal}\left(\overline{\mathbb{Q}}_{p} / L\right) \rightarrow \mathrm{GL}_{2}\left(\overline{\mathbb{F}}_{p}\right)$ une représentation continue réductible et suffisamment générique. Suivant la démarche de [6], on montre comment relier le type d'extension de $\bar{\rho}$ à certains paramètres définissant les diagrammes de Diamond associés à $\bar{\rho}$.

Mots-clefs : Correspondance de Langlands $\bmod p$, poids de Serre, diagrammes de Diamond.

A bstract - Special VAlues of PARAMETERS in DiAmond DiAgRAMS - Let $L$ be a finite unramified extension of $\mathbb{Q}_{p}$ and $\bar{\rho}: \operatorname{Gal}\left(\overline{\mathbb{Q}}_{p} / L\right) \rightarrow \mathrm{GL}_{2}\left(\overline{\mathbb{F}}_{p}\right)$ be a reducible continuous generic representation. Following the strategy in [6], we show how the extension type of $\bar{\rho}$ is related to certain parameters which appear in the Diamond diagrams associated to $\bar{\rho}$.

Keywords : Mod $p$ Langlands correspondence, Serre weights, Diamond diagrams.

Class. math. : 22E50, 11F85, 11F70.

\section{Table des matières}

1 Introduction 1

2 Rappels sur les diagrammes de Diamond 4

3 Modules fortement divisibles et leurs réductions $\quad 10$

4 Valeurs spéciales de paramètres $\quad 15$

5 Paramètres cycliques $\quad 21$

6 Nombre de paramètres 28

\section{Introduction}

Soient $p$ un nombre premier et $L$ une extension finie de $\mathbb{Q}_{p}$ avec $\mathcal{O}_{L}$ son anneau des entiers. La correspondance de Langlands locale modulo $p$ pour $\mathrm{GL}_{2}\left(\mathbb{Q}_{p}\right)$ est maintenant bien comprise ([1], [2], [11]), mais celle pour $\mathrm{GL}_{2}(L)$ lorsque $L \neq \mathbb{Q}_{p}$ reste encore largement mystérieuse. Dans ce cas, l'un des principaux obstacles est l'existence de beaucoup plus de représentations (modulo $p$ ou $p$-adiques) de $\mathrm{GL}_{2}(L)$ que de représentations de dimension 2 (modulo $p$ ou $p$-adiques) de $\operatorname{Gal}\left(\overline{\mathbb{Q}}_{p} / L\right)$. Par exemple, lorsque $L$ est non ramifiée sur $\mathbb{Q}_{p}$, inspirés par les travaux de Buzzard, Diamond et Jarvis ([9]), Breuil et Paškūnas ont construit des représentations lisses admissibles sur $\overline{\mathbb{F}}_{p}$ de $\mathrm{GL}_{2}(L)$ satisfaisant une certaine condition sur les $\mathrm{GL}_{2}\left(\mathcal{O}_{L}\right)$-socles (voir [8]). Mais cela est loin de permettre d'isoler une unique représentation, comme démontre l'auteur [13]. 
Pourtant, en fixant une représentation continue de $\operatorname{Gal}\left(\overline{\mathbb{Q}}_{p} / L\right)$ de dimension 2 sur $\overline{\mathbb{F}}_{p}$, on espère pouvoir ajouter de "bonnes" conditions restrictives du coté $\mathrm{GL}_{2}(L)$ à celle portant sur le socle, pour améliorer la sélection. Jusqu'à présent (à la connaissance de l'auteur), les conditions exhibées vivent toutes dans les diagrammes de Diamond associés à la représentation galoisienne (donc toujours pour $L$ non ramifiée). La première condition de ce genre a été définie dans [4], motivée par la compatibilité souhaitée avec un possible foncteur de Colmez généralisé ([11],[17]).

Une deuxième condition est considérée dans [6], et fait aussi l'objet du présent article. Pour expliquer plus clairement notre situation, nous rappelons brièvement la construction de diagrammes de Diamond d'après [8]. Supposons $L$ non ramifiée sur $\mathbb{Q}_{p}$ et fixons une représentation continue $\bar{\rho}: \operatorname{Gal}\left(\overline{\mathbb{Q}}_{p} / L\right) \rightarrow \mathrm{GL}_{2}\left(\overline{\mathbb{F}}_{p}\right)$ que l'on suppose suffisamment générique (à préciser dans le texte). A $\bar{\rho}$ sont associés un ensemble de poids de Serre noté $\mathcal{D}(\bar{\rho})$ ainsi qu'une $\mathrm{GL}_{2}\left(\mathcal{O}_{L}\right)$-représentation $D(\bar{\rho})$ de dimension finie sur $\overline{\mathbb{F}}_{p}$ dont le socle est exactement $\oplus_{\tau \in \mathcal{D}(\bar{\rho})} \tau$ et qui est maximale pour une certaine condition de multiplicité 1 . Notons $I\left(\mathcal{O}_{L}\right)$ le sous-groupe d'Iwahori de $\mathrm{GL}_{2}\left(\mathcal{O}_{L}\right)$ et $I_{1}\left(\mathcal{O}_{L}\right)$ le pro-p-sous-groupe de $I\left(\mathcal{O}_{L}\right)$. En tant que $I\left(\mathcal{O}_{L}\right)$-représentation, l'espace des $I_{1}\left(\mathcal{O}_{L}\right)$-invariants de $D(\bar{\rho})$ est une somme directe de caractères qui apparaissent par paires stables sous la conjugaison par $\left(\begin{array}{ll}0 & 1 \\ p & 0\end{array}\right)$. On peut donc y définir une action de $\left(\begin{array}{ll}0 & 1 \\ p & 0\end{array}\right)$, mais elle n'est pas unique a priori. Un diagramme de Diamond associé à $\bar{\rho}$ est alors simplement l'injection naturelle $D(\bar{\rho})^{I_{1}\left(\mathcal{O}_{L}\right)} \hookrightarrow D(\bar{\rho})$, pour une action de $\left(\begin{array}{ll}0 & 1 \\ p & 0\end{array}\right)$ sur $D(\bar{\rho})^{I_{1}\left(\mathcal{O}_{L}\right)}$ choisie.

On peut classifier les actions possibles de $\left(\begin{array}{ll}0 & 1 \\ p & 0\end{array}\right)$ sur $D(\bar{\rho})^{I_{1}\left(\mathcal{O}_{L}\right)}$ (à isomorphisme près) à l'aide de certains paramètres (voir $\S 6$ ). Mais dès que $\bar{\rho}$ est réductible non scindée, la donnée de $\mathcal{D}(\bar{\rho})$, ou de $D(\bar{\rho})$, contient moins d'informations que la représentation $\bar{\rho}$ de départ. Nous voulons donc relier la classe d'isomorphisme de $\bar{\rho}$ aux paramètres définissant l'action de $\left(\begin{array}{ll}0 & 1 \\ p & 0\end{array}\right)$ sur $D(\bar{\rho})^{I_{1}\left(\mathcal{O}_{L}\right)}$.

Décrivons les paramètres qui nous intéressent dans cet article. Rappelons que $D(\bar{\rho})$ se décompose sous la forme $\bigoplus_{\tau \in \mathcal{D}(\bar{\rho})} D_{\tau}(\bar{\rho})$, où $D_{\tau}(\bar{\rho})$ est l'unique facteur direct de $D(\bar{\rho})$ de socle $\tau$. Soit $\chi$ un caractère de $I\left(\mathcal{O}_{L}\right)$ apparaissant sur $D_{\tau}(\bar{\rho})^{I_{1}\left(\mathcal{O}_{L}\right)}$ et soit $v \in D_{\tau}(\bar{\rho})^{I_{1}\left(\mathcal{O}_{L}\right)}$ un vecteur propre de caractère $\chi$. Sous l'action d'un élément dans $\mathrm{GL}_{2}\left(\mathcal{O}_{L}\right)$ d'une forme particulière mais explicite (voir la proposition 2.6), $v$ engendre un vecteur dans $\tau^{I_{1}} \hookrightarrow$ $D_{\tau}(\bar{\rho})^{I_{1}\left(\mathcal{O}_{L}\right)}$, que l'on note $w_{1}$ pour l'instant. Si le conjugué $\chi^{s}$ de $\chi$ par $\left(\begin{array}{ll}0 & 1 \\ p & 0\end{array}\right)$ apparaît aussi sur $D_{\tau}(\bar{\rho})^{I_{1}\left(\mathcal{O}_{L}\right)}$, on obtient un autre vecteur non nul $w_{2}$ de $\tau^{I_{1}}$ à partir de $\left(\begin{array}{ll}0 & 1 \\ p & 0\end{array}\right) v$. Comme $\tau^{I_{1}}$ est de dimension 1 sur $\overline{\mathbb{F}}_{p}, w_{1}$ et $w_{2}$ diffèrent par un scalaire non nul $x(\chi) \in \overline{\mathbb{F}}_{p}^{\times}$, dont on vérifie qu'il ne dépend pas du choix de $v$. C'est par définition le paramètre associé à $\chi$. En général, il existe des diagrammes de Diamond avec $x(\chi)$ prenant n'importe quelle valeur de $\overline{\mathbb{F}}_{p}^{\times}$. Dans [6], lorsque $\chi$ et $\chi^{s}$ apparaissent tous les deux sur la "première" composante $D_{\tau(\emptyset)}(\bar{\rho})^{I_{1}\left(\mathcal{O}_{L}\right)}$ de $D(\bar{\rho})^{I_{1}\left(\mathcal{O}_{L}\right)}$, une valeur de $x(\chi)$ d'intérêt "particulier" est isolée. Dans l'article présent, nous traitons le cas général en suivant la démarche de [6]. On définit aussi des paramètres cycliques comme dans [4] et l'on montre que si $|\mathcal{D}(\bar{\rho})|=1$, les valeurs spéciales que l'on trouve sont alors exactement celles prédites dans $[4, \S 6]$.

Les énoncés des résultats principaux de l'article nécessitant l'introduction de trop nombreuses de notations, nous préférons de ne pas les rappeler dans cette introduction mais renvoyer le lecteur aux théorèmes 4.7 et 5.6 pour plus de détails. Expliquons quand même d'où vient l'idée. La représentation $\bar{\rho}$ admet plusieurs déformations potentiellement Barsotti-Tate qui deviennent cristallines sur une extension modérément ramifiée de $L$ et de type d'inertie associé à $\chi$, donc correspondant par la correspondance de Langlands locale classique à des séries principales modérément ramifiées de $\mathrm{GL}_{2}(L)$ sur $\overline{\mathbb{Q}}_{p}$. Soit $\Pi$ une telle série principale. 
En admettant l'existence d'une compatibilité local-global modulo $p$ pour $\mathrm{GL}_{2}(L)$, la donnée de $\bar{\rho}$ devrait correspondre à celle de la réduction d'un certain réseau $\mathrm{GL}_{2}(L)$-invariant de $\Pi$. En général, les réseaux $\mathrm{GL}_{2}(L)$-invariants de $\Pi$ (lorsqu'ils existent) sont très difficiles à décrire, mais nous n'avons heureusement pas besoin ici de les décrire explicitement, et le paramètre qui nous intéresse ne dépend pas du choix du réseau, sous réserve qu'il se trouve dans la réduction modulo $p$ dudit réseau.

Le plan de l'article est le suivant. Au $\S 2$, on fait quelques rappels et l'on démontre la proposition 2.1, dans laquelle on détermine une condition exacte sur $\chi \in D(\bar{\rho})^{I_{1}\left(\mathcal{O}_{L}\right)}$ pour que le paramètre associé soit bien défini. Au $\S 3$, on calcule la représentation galoisienne résiduelle associée à un module fortement divisible avec donnée de descente modérément ramifiée. Cette partie est essentiellement une relecture des calculs effectués dans [5]. On démontre le résultat principal de cet article au $\S 4$. Au $\S 5$, on définit et calcule des paramètres cycliques analogues à ceux définis dans [4]. Enfin au $§ 6$, on détermine le nombre de paramètres nécessaires à classifier les diagrammes de Diamond associés à $\bar{\rho}$.

Introduisons les notations générales de l'article.

Dans tout le texte, $L$ désigne une extension non ramifiée de $\mathbb{Q}_{p}$ de degré $f$, d'anneau des entiers $\mathcal{O}_{L}$ et $E$ désigne une extension finie de $\mathbb{Q}_{p}$ (qui sera le corps des coefficients) d'anneau des entiers $\mathcal{O}_{E}$ et de corps résiduel $k_{E}$. On suppose que $E$ est suffisamment grand afin d'avoir $\left|\operatorname{Hom}_{\mathbb{Q}_{p}}(L, E)\right|=f$. On note $q=p^{f}$ et l'on identifie le corps résiduel de $L$ avec $\mathbb{F}_{q}$. On note $\varphi: \mathbb{F}_{q} \rightarrow \mathbb{F}_{q}$ le Frobenius $x \mapsto x^{p}$. On normalise la valuation $p$-adique val sur $L$ ou $E \operatorname{par} \operatorname{val}(p)=1$ et on pose $|\cdot|=\frac{1}{q^{\text {val(.) }}}$.

On fixe une fois pour toutes un plongement $\sigma_{0}$ de $\mathbb{F}_{q}$ dans $k_{E}$, de sorte que $\left\{\sigma_{0} \circ\right.$ $\left.\varphi^{i}\right\}_{0 \leq i \leq f-1}$ sont les $f$ plongements distincts de $\mathbb{F}_{q}$ dans $k_{E}$. On normalise l'application de réciprocité de la théorie du corps de classes local en envoyant les Frobenius géométriques sur les uniformisantes, via laquelle on voit tout caractère de $L^{\times}$comme un caractère de $\operatorname{Gal}\left(\overline{\mathbb{Q}}_{p} / L\right)$.

On a de plus un isomorphisme canonique :

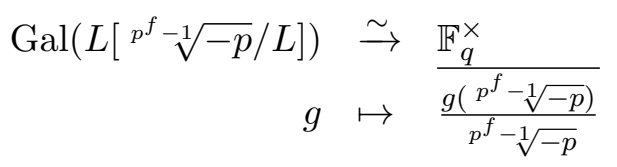

via lequel on peut voir implicitement tout caractère de $\mathbb{F}_{q}^{\times}$comme un caractère du groupe $\operatorname{Gal}\left(L\left[p^{f}-1 /-p\right] / L\right)$ ou du groupe $\operatorname{Gal}\left(\overline{\mathbb{Q}}_{p} / L\right)$. On note $\omega_{\sigma_{0}}: \operatorname{Gal}\left(\overline{\mathbb{Q}}_{p} / L\right) \rightarrow \mathbb{F}_{q}^{\times} \stackrel{\sigma_{0}}{\hookrightarrow} k_{E}^{\times}$le caractère induit, $\omega$ le caractère cyclotomique modulo $p$ (via $\mathbb{F}_{p} \hookrightarrow k_{E}$ ) et $\operatorname{nr}(x)$ le caractère non ramifié de $L^{\times}$envoyant $p$ sur $x$ (pour $x \in k_{E}^{\times}$ou $E^{\times}$).

On rappelle qu'un poids de Serre, ou simplement un poids, est une représentation absolument irréductible de $\mathrm{GL}_{2}\left(\mathcal{O}_{L}\right)$, ou de manière équivalente de $\mathrm{GL}_{2}\left(\mathbb{F}_{q}\right)$, sur $k_{E}$. À isomorphisme près, tout poids est de la forme

$$
\left(\otimes_{i=0}^{f-1}\left(\operatorname{Sym}^{r_{i}} k_{E}^{2}\right)^{\sigma_{0} \circ \varphi^{i}}\right) \otimes \operatorname{det}^{a},
$$

avec $r_{i} \in\{0, \cdots, p-1\}, a \in\{0, \cdots, q-2\}$, et où $\mathrm{GL}_{2}\left(\mathbb{F}_{q}\right)$ agit sur $\left(\mathrm{Sym}^{r_{i}} k_{E}^{2}\right)^{\sigma_{0} \circ \varphi^{i}}$ via $\sigma_{0} \circ \varphi^{i}: \mathbb{F}_{q} \hookrightarrow k_{E}$ et via l'action naturelle de $\mathrm{GL}_{2}\left(k_{E}\right)$ sur la base canonique de $k_{E}^{2}$. On désignera par $\left(r_{0}, \cdots, r_{f-1}\right) \otimes \operatorname{det}^{a}$ cette représentation.

On note $\mathcal{S}$ l'ensemble $\{0, \cdots, f-1\}$ et on définit une application bijective $\delta$ de l'ensemble des parties $J$ de $\mathcal{S}$ dans lui-même en posant $j \in \delta(J)$ si et seulement si $j+1 \in J$. 
On note $B(L) \subset \mathrm{GL}_{2}(L)$ le sous-groupe des matrices triangulaires supérieures et $I\left(\mathcal{O}_{L}\right) \subset$ $\mathrm{GL}_{2}\left(\mathcal{O}_{L}\right)$ (resp. $I_{1}\left(\mathcal{O}_{L}\right) \subset \mathrm{GL}_{2}\left(\mathcal{O}_{L}\right)$ ) le sous-groupe des matrices triangulaires supérieures (resp. et unipotents) modulo $p$. Si $\chi$ est un caractère lisse de $I\left(\mathcal{O}_{L}\right)$ à valeurs dans $k_{E}^{\times}$, on note $\chi^{s}$ le caractère conjugué de $\chi$ par $\left(\begin{array}{ll}0 & 1 \\ p & 0\end{array}\right)$, i.e. $\chi^{s}\left(\left(\begin{array}{cc}a & b \\ p c & d\end{array}\right)\right):=\chi\left(\left(\begin{array}{cc}d & c \\ p b & a\end{array}\right)\right)$ si $\left(\begin{array}{cc}a & b \\ p c & d\end{array}\right) \in I\left(\mathcal{O}_{L}\right)$. On note $\bar{\sigma}(\chi)$ le $k_{E}$-espace vectoriel $\operatorname{Ind}_{I\left(\mathcal{O}_{L}\right)}^{\mathrm{GL}_{2}\left(\mathcal{O}_{L}\right)} \chi$ des fonctions $f: \mathrm{GL}_{2}\left(\mathcal{O}_{L}\right) \rightarrow k_{E}$ telles que $f\left(g g^{\prime}\right)=\chi(g) f\left(g^{\prime}\right)$ (pour $g \in I\left(\mathcal{O}_{L}\right)$ et $g^{\prime} \in \mathrm{GL}_{2}\left(\mathcal{O}_{L}\right)$ ) muni de l'action à gauche de $\mathrm{GL}_{2}\left(\mathcal{O}_{L}\right)$ par translation à droite. Notons que cette action se factorise par $\mathrm{GL}_{2}\left(\mathbb{F}_{q}\right)$, car $\chi$

est toujours trivial sur $I_{1}\left(\mathcal{O}_{L}\right)$. On définit de même l'induite $\operatorname{Ind}_{B(L)}^{\mathrm{GL}_{2}(L)} \chi$ pour un caractère lisse $\chi$ de $B(L)$ dans $E^{\times}$.

Remarquons enfin que même si cet article est une continuation de [6], on suivra plutôt les notations de [5].

Remerciements : Je remercie Christophe Breuil pour avoir porté ce problème à mon attention et pour ses nombreuses suggestions. Je remercie l'institut Max-Planck de Mathématiques et le centre BICMR à Pékin pour leur accueil durant la rédaction de l'article. Je remercie également le rapporteur anonyme pour sa lecture minutieuse et ses remarques pertinentes.

\section{Rappels sur les diagrammes de Diamond}

dans cette section, on fait des rappels de [8] et [5] sur les diagrammes de Diamond et l'on établit certains résultats combinatoires qui seront utilisés dans la suite.

On fixe une représentation continue réductible $\bar{\rho}: \operatorname{Gal}\left(\overline{\mathbb{Q}}_{p} / L\right) \rightarrow \mathrm{GL}_{2}\left(k_{E}\right)$ de la forme

$$
\bar{\rho}=\left(\begin{array}{cc}
\operatorname{nr}(\xi) \omega_{\sigma_{0}}^{\sum_{j=0}^{f-1} p^{j}\left(r_{j}+1\right)} & * \\
0 & \operatorname{nr}\left(\xi^{-1}\right)
\end{array}\right),
$$

avec $\xi \in k_{E}^{\times}, r_{j} \in\{0, \cdots, p-3\}$ et $\left(r_{j}\right)_{j} \notin\{(0, \cdots, 0),(p-3, \cdots, p-3)\}$, cette dernière condition étant la "généricité" introduite dans $[8, \S 11]$. À $\bar{\rho}$ est associé dans [9] un ensemble $\mathcal{D}(\bar{\rho})$ de poids de Serre. Il admet une description explicite à l'aide d'un certain sous-ensemble de $\{0, \cdots, f-1\}$ dont on rappelle maintenant la construction suivant la reformulation dans [5].

Grâce à la condition de généricité, $\bar{\rho}$ est dans la catégorie de Fontaine-Laffaille [12], donc s'écrit sous la forme

$$
\bar{\rho}=\operatorname{Hom}_{\mathrm{Fil}^{\cdot}, \varphi \cdot}\left(M, A_{\text {cris }} \otimes_{\mathbb{Z}_{p}} \mathbb{F}_{p}\right)
$$

où $M$ est un $\varphi$-module filtré de Fontaine-Laffaille uniquement déterminé par $\bar{\rho}$ tandis que $A_{\text {cris }}$ est l'anneau de périodes de Fontaine pour les représentations cristallines entières et $\mathrm{Hom}_{\mathrm{Fil}, \varphi}$, indique que l'on considère les morphismes qui respectent les filtrations et qui commutent à $\varphi$ (cf. [12] pour les détails). On peut exprimer $M$ sous la forme suivante [6, $\S 2.1]$

$$
M=M^{\sigma_{0}} \times \cdots \times M^{\sigma_{0} \circ \varphi^{-(f-1)}}, \quad \text { avec } M^{\sigma_{0} \circ \varphi^{-j}}=k_{E} e^{\sigma_{0} \circ \varphi^{-j}} \oplus k_{E} f^{\sigma_{0} \circ \varphi^{-j}}
$$

muni de la filtration suivante, où l'on pose $M^{j}=M^{\sigma_{0} \circ \varphi^{-j}}, e^{j}=e^{\sigma_{0} \circ \varphi^{-j}}$ et $f^{j}=f^{\sigma_{0} \circ \varphi^{-j}}$ 
pour alléger les notations et où l'on convient que $(f-1)+1=0$ :

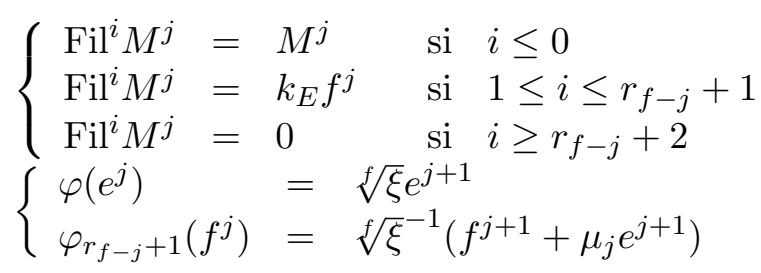

On a noté $\sqrt[f]{\xi}$ une racine $f$-ième fixée $\xi$ dans $k_{E}$, dont on peut supposer l'existence quitte à agrandir $E$ et $\mu_{j} \in k_{E}$ pour tout $j$. Les choix faits ci-dessus pour $M$ ne sont pas uniques, mais on vérifie que l'ensemble $J_{\bar{\rho}}$ défini par

$$
J_{\bar{\rho}}:=\left\{j \in\{0, \cdots, f-1\}, \mu_{f-j}=0\right\}
$$

ne dépend que de $\bar{\rho}$. (Cet ensemble est noté par $Z(\bar{\rho})$ dans [6].) De plus, on vérifie que $J_{\bar{\rho}}=\mathcal{S}$ si et seulement si $\bar{\rho}$ est scindée.

On dispose de la description explicite suivante de l'ensemble $\mathcal{D}(\bar{\rho})$. Soit $\left(x_{0}, \cdots, x_{f-1}\right)$ $f$ variables. On note $\mathcal{D}\left(x_{0}, \cdots, x_{f-1}\right)$ l'ensemble des $f$-uplets $\tau=\left(\tau_{0}\left(x_{0}\right), \cdots, \tau_{f-1}\left(x_{f-1}\right)\right)$ vérifiant les conditions suivantes :

(i) $\tau_{i}\left(x_{i}\right) \in\left\{x_{i}, x_{i}+1, p-2-x_{i}, p-3-x_{i}\right\}$

(ii) si $\tau_{i}\left(x_{i}\right) \in\left\{x_{i}, x_{i}+1\right\}$, alors $\tau_{i+1}\left(x_{i+1}\right) \in\left\{x_{i+1}, p-2-x_{i+1}\right\}$

(iii) si $\tau_{i}\left(x_{i}\right) \in\left\{p-2-x_{i}, p-3-x_{i}\right\}$, alors $\tau_{i+1}\left(x_{i+1}\right) \in\left\{p-3-x_{i+1}, x_{i+1}+1\right\}$

(iv) $\tau_{i}\left(x_{i}\right) \in\left\{p-3-x_{i}, x_{i}+1\right\}$ implique $i \in J_{\bar{\rho}}$

où l'on convient que $x_{f}=x_{0}$ et $\tau_{f}\left(x_{f}\right)=\tau_{0}\left(x_{0}\right)$. Notons que les conditions (i)-(iii) entraînent $\tau_{0}\left(x_{0}\right) \in\left\{x_{0}, p-3-x_{0}\right\}$ si $f=1$. Avec ces notations, on a la description suivante pour $\mathcal{D}(\bar{\rho})$ (voir [5, Prop. A.3] ou [10]) :

$$
\mathcal{D}(\bar{\rho})=\left\{\left(\tau_{0}\left(r_{0}\right), \cdots, \tau_{f-1}\left(r_{f-1}\right)\right) \otimes \operatorname{det}^{e(\tau)\left(r_{0}, \cdots, r_{f-1}\right)}, \tau \in \mathcal{D}\left(x_{0}, \cdots, x_{f-1}\right)\right\},
$$

où $e(\tau)\left(x_{0}, \cdots, x_{f-1}\right)$ est défini comme dans $[5, \S 4]$ que l'on ne précise pas ici. On remarque que cela définit une bijection entre $\mathcal{D}\left(x_{0}, \cdots, x_{f-1}\right)$ et $\mathcal{D}(\bar{\rho})$ : en effet, si $\tau \neq \tau^{\prime}$ sont tels que $\tau_{i}\left(r_{i}\right)=\tau_{i}^{\prime}\left(r_{i}\right)$ pour tout $i$, on aura $e(\tau)\left(r_{0}, \cdots, r_{f-1}\right) \not \equiv e\left(\tau^{\prime}\right)\left(r_{0}, \cdots, r_{f-1}\right) \bmod (q-1)$ (cf. la preuve de [8, Lem.12.8]). Dans la suite, on notera encore $\tau$ le poids associé au $f$-uplet $\tau=\left(\tau_{i}\left(x_{i}\right)\right)_{i}$ défini dans $(3)$.

Notons $\bar{\rho}^{\text {ss }}$ la semi-simplification de $\bar{\rho}$ : on a alors $J_{\bar{\rho}^{\mathrm{ss}}}=\mathcal{S}$ et la construction ci-dessus montre que $\mathcal{D}\left(\bar{\rho}^{\mathrm{ss}}\right)$ consiste en les poids $\tau$ vérifiant les conditions (i)-(iii), la (iv) étant automatique. En particulier, on a $\mathcal{D}(\bar{\rho}) \subset \mathcal{D}\left(\bar{\rho}^{\mathrm{ss}}\right)$. Ceci nous mène à poser, pour tout $\tau \in$ $\mathcal{D}\left(\bar{\rho}^{\mathrm{ss}}\right)$ :

$$
J_{\tau}:=\left\{i \in\{0, \cdots, f-1\}, \tau_{i}\left(x_{i}\right) \in\left\{x_{i}+1, p-3-x_{i}\right\}\right\} \subseteq \mathcal{S},
$$

et permet d'identifier $\mathcal{D}\left(\bar{\rho}^{\text {ss }}\right)$ (resp. $\left.\mathcal{D}(\bar{\rho})\right)$ à l'ensemble des parties de $\mathcal{S}$ (resp. $\left.J_{\bar{\rho}}\right)$ via $\tau \mapsto J_{\tau}$.

Soit $D(\bar{\rho})$ la représentation maximale (pour l'inclusion) de $\mathrm{GL}_{2}\left(\mathbb{F}_{q}\right)$ sur $k_{E}$ vérifiant les deux conditions suivantes :

(i) le $\mathrm{GL}_{2}\left(\mathbb{F}_{q}\right)$-socle (i.e. la sous-représentation semi-simple maximale) de $D(\bar{\rho})$ est $\oplus_{\tau \in \mathcal{D}(\bar{\rho})} \tau$;

(ii) chaque poids $\tau \in \mathcal{D}(\bar{\rho})$ n'apparaît qu'une seule fois dans $D(\bar{\rho})$. 
L'existence et l'unicité d'une telle représentation sont montrées dans [8, §13], où elle est notée $D_{0}(\bar{\rho})$, et on peut naturellement la voir comme une représentation de $\mathrm{GL}_{2}\left(\mathcal{O}_{L}\right) L^{\times}$ en y faisant agir $p$ trivialement. De plus, on sait que $D(\bar{\rho})$ se décompose sous la forme $\oplus_{\tau \in \mathcal{D}(\bar{\rho})} D_{\tau}(\bar{\rho})$ où $D_{\tau}(\bar{\rho})$ est l'unique facteur direct de $D(\bar{\rho})$ de socle égal à $\tau$. Les caractères de $I\left(\mathcal{O}_{L}\right)$ qui apparaissent sur $D(\bar{\rho})^{I_{1}\left(\mathcal{O}_{L}\right)}$ sont en outre tous distincts [8, Thm. 13.8] et sont caractérisés par un ensemble de $f$-uplets noté $\mathcal{P D}\left(x_{0}, \cdots, x_{f-1}\right)$ qui est défini comme suit. L'ensemble $\mathcal{P D}\left(x_{0}, \cdots, x_{f-1}\right)$ est constitué des $f$-uplets $\lambda=\left(\lambda_{i}\left(x_{i}\right)\right)_{i}$ tels que :

(i) $\lambda_{i}\left(x_{i}\right) \in\left\{x_{i}, x_{i}+1, x_{i}+2, p-3-x_{i}, p-2-x_{i}, p-1-x_{i}\right\}$

(ii) si $\lambda_{i}\left(x_{i}\right) \in\left\{x_{i}, x_{i}+1, x_{i}+2\right\}$, alors $\lambda_{i+1}\left(x_{i+1}\right) \in\left\{x_{i+1}, x_{i+1}+2, p-2-x_{i+1}\right\}$

(iii) si $\lambda_{i}\left(x_{i}\right) \in\left\{p-1-x_{i}, p-2-x_{i}, p-3-x_{i}\right\}$, alors $\lambda_{i+1}\left(x_{i+1}\right) \in\left\{p-1-x_{i+1}, p-3-\right.$ $\left.x_{i+1}, x_{i+1}+1\right\}$

(iv) $\lambda_{i}\left(x_{i}\right) \in\left\{p-3-x_{i}, x_{i}+2\right\}$ implique $i \in J_{\bar{\rho}}$.

On convient bien sûr comme avant que $x_{f}=x_{0}$ et $\lambda_{f}\left(x_{f}\right)=\lambda_{0}\left(x_{0}\right)$. On pose alors, pour tout $\lambda \in \mathcal{P} \mathcal{D}\left(x_{0}, \cdots, x_{f-1}\right)$,

$$
\tau(\lambda):=\left(\lambda_{0}\left(r_{0}\right), \cdots, \lambda_{f-1}\left(r_{f-1}\right)\right) \otimes \operatorname{det}^{e(\lambda)\left(r_{0}, \cdots, r_{f-1}\right)}
$$

où $e(\lambda)\left(x_{0}, \cdots, x_{f-1}\right)$ est défini comme dans [5, §4]. On vérifie que $\tau(\lambda) \varsubsetneqq \tau\left(\lambda^{\prime}\right)$ pour tous $f$-uplets distincts $\lambda \neq \lambda^{\prime}$ de $\mathcal{P D}\left(x_{0}, \cdots, x_{f-1}\right)$. D'après [5, Prop. 4.2], les caractères de $I\left(\mathcal{O}_{L}\right)$ qui apparaissent sur $D(\bar{\rho})^{I_{1}\left(\mathcal{O}_{L}\right)}$ sont exactement les caractères $\tau(\lambda)^{I_{1}\left(\mathcal{O}_{L}\right)}$ avec $\lambda \in$ $\mathcal{P D}\left(x_{0}, \cdots, x_{f-1}\right)$. On remarque que si $\chi$ correspond à $\lambda$, alors son conjugué $\chi^{s}$ correspond à $\lambda^{[s]}$ où $\lambda^{[s]} \in \mathcal{P D}\left(x_{0}, \cdots, x_{f-1}\right)$ est défini par $\lambda_{i}^{[s]}\left(x_{i}\right)=p-1-\lambda_{i}\left(x_{i}\right)$. En particulier $\chi \neq \chi^{s}$ et $D(\bar{\rho})^{I_{1}\left(\mathcal{O}_{L}\right)}$ est stable par la transformation $\chi \mapsto \chi^{s}$.

Soit maintenant $\chi: I\left(\mathcal{O}_{L}\right) \rightarrow k_{E}^{\times}$un caractère apparaissant sur $D(\bar{\rho})^{I_{1}\left(\mathcal{O}_{L}\right)}$ et soit $\lambda \in \mathcal{P D}\left(x_{0}, \cdots, x_{f-1}\right)$ le $f$-uplet associé. Comme $\chi^{s}$ apparaît aussi sur $D(\bar{\rho})^{I_{1}\left(\mathcal{O}_{L}\right)}$ par ce qui précède, il existe par réciprocité de Frobenius un morphisme $\mathrm{GL}_{2}\left(\mathcal{O}_{L}\right)$-équivariant non nul de $\bar{\sigma}\left(\chi^{s}\right):=\operatorname{Ind}_{I\left(\mathcal{O}_{L}\right)}^{\mathrm{GL}_{2}\left(\mathcal{O}_{L}\right)} \chi^{s}$ vers $D(\bar{\rho})$, de sorte que $\bar{\sigma}\left(\chi^{s}\right)$ admet au moins un élément de $\mathcal{D}(\bar{\rho})$ comme sous-quotient. D'ailleurs, les constituants de $\bar{\sigma}\left(\chi^{s}\right)$ sont naturellement indexés par un certain sous-ensemble $\mathcal{P}_{\chi}$ de l'ensemble des parties de $\mathcal{S}=\{0, \cdots, f-1\}$. On renvoie le lecteur à $[5, \S 2]$ pour la définition précise de $\mathcal{P}_{\chi}$, en rappelant seulement que $\mathcal{P}_{\chi}$ contient toujours $\{\emptyset, \mathcal{S}\}$ et que le socle de $\bar{\sigma}\left(\chi^{S}\right)$, à savoir $\tau(\lambda)$, est indexé par $\emptyset$ tandis que le cosocle est indexé par $\mathcal{S}$. D'après [5, Prop. 4.3], il existe un unique couple $\left(J^{\min }, J^{\max }\right) \in \mathcal{P}_{\chi} \times \mathcal{P}_{\chi}$ vérifiant $J^{\min } \subseteq J^{\max }$ et $J^{\max } \backslash J^{\min } \subseteq \delta\left(J_{\bar{\rho}}\right)$ tel que les facteurs de Jordan-Hölder de $\bar{\sigma}\left(\chi^{s}\right)$ qui sont des poids de Serre pour $\bar{\rho}$ soient exactement les poids indexés par les parties de $\mathcal{S}$ contenant $J^{\min }$ et contenues dans $J^{\max }$. Explicitement, $J^{\min }$ et $J^{\max }$ sont définies comme suit $[5,(19)]$ :

$$
\begin{aligned}
& J^{\min }=\delta\left(\left\{j \mid \lambda_{j}\left(x_{j}\right) \in\left\{p-1-x_{j}, x_{j}+2\right\} \text { ou }\left(\lambda_{j}\left(x_{j}\right)=x_{j}+1 \text { et } j \notin J_{\bar{\rho}}\right)\right\}\right) ; \\
& J^{\max }=\delta\left(\left\{j \mid \lambda_{j}\left(x_{j}\right) \notin\left\{p-3-x_{j}, x_{j}\right\} \text { et }\left(j \in J_{\bar{\rho}} \text { si } \lambda_{j}\left(x_{j}\right)=p-2-x_{j}\right)\right\}\right) .
\end{aligned}
$$

On en déduit que si $\tau \in \mathcal{D}(\bar{\rho})$ est l'unique élément tel que $\chi^{s}$ apparaît sur $D_{\tau}(\bar{\rho})^{I_{1}\left(\mathcal{O}_{L}\right)}$, alors $\tau$ est exactement le sous-quotient de $\bar{\sigma}\left(\chi^{s}\right)$ indexé par $J^{\max }$ (par construction de $D(\bar{\rho})$ ). Le résultat suivant détermine toutes les paires $\left\{\chi, \chi^{s}\right\}$ qui apparaissent sur le même facteur $D_{\tau}(\bar{\rho})^{I_{1}\left(\mathcal{O}_{L}\right)}$. 
Proposition 2.1. Conservons les notations précédentes. Pour que les caractères $\chi$ et $\chi^{s}$ apparaissent sur le même facteur $D_{\tau}(\bar{\rho})^{I_{1}\left(\mathcal{O}_{L}\right)}$ avec $\tau \in \mathcal{D}(\bar{\rho})$, il faut et il suffit que l'on ait

$$
J_{\bar{\rho}} \cap\left\{j \mid \lambda_{j}\left(x_{j}\right) \in\left\{x_{j}+1, p-2-x_{j}\right\}\right\}=\emptyset .
$$

Dans ce cas, on a $J_{\tau}=\left\{j \mid \lambda_{j}\left(x_{j}\right) \in\left\{p-3-x_{j}, x_{j}+2\right\}\right\}$.

Démonstration. Soient respectivement $\tau$ et $\tau^{\prime}$ les poids de $\mathcal{D}(\bar{\rho})$ tels que $\chi^{s}$ apparaisse sur $D_{\tau}(\bar{\rho})^{I_{1}\left(\mathcal{O}_{L}\right)}$ et $\chi$ sur $D_{\tau^{\prime}}(\bar{\rho})^{I_{1}\left(\mathcal{O}_{L}\right)}$. Par ce qui précède, $\tau$ et $\tau^{\prime}$ sont tous deux des sousquotients de $\bar{\sigma}\left(\chi^{s}\right)$ car $\bar{\sigma}(\chi)$ et $\bar{\sigma}\left(\chi^{s}\right)$ ont les mêmes facteurs de Jordan-Hölder, et $\tau$ y est indexé par $J^{\max }$ tandis que $\tau^{\prime}$ l'est par $J^{\min }$. Par conséquent, on a $\tau=\tau^{\prime}$ si et seulement si $J^{\min }=J^{\max }$, ce qui est équivalent à la condition (6) de l'énoncé par examen des définitions de $J^{\max }$ et de $J^{\text {min }}$.

Supposons maintenant que la condition (6) soit satisfaite et déterminons $J_{\tau}$. Par ce qui précède, $\tau$ est le sous-quotient de $\bar{\sigma}\left(\chi^{s}\right)$ indexé par :

$$
J^{\min }=J^{\max }=\delta\left(\left\{j \mid \lambda_{j}\left(x_{j}\right) \in\left\{p-1-x_{j}, x_{j}+1, x_{j}+2\right\}\right\}\right) .
$$

Connaissant la structure de $\bar{\sigma}\left(\chi^{s}\right)$ décrite dans [8, §2], on peut trouver un unique $f$-uplet $\mu=\left(\mu_{0}\left(y_{0}\right), \cdots, \mu_{f-1}\left(y_{f-1}\right)\right)$ vérifiant les trois conditions suivantes :

(a) $\mu_{i}\left(y_{i}\right) \in\left\{y_{i}, y_{i}-1, p-2-y_{i}, p-1-y_{i}\right\}$

(b) $\mu_{i}\left(y_{i}\right) \in\left\{y_{i}, y_{i}-1\right\}$ implique $\mu_{i+1}\left(y_{i+1}\right) \in\left\{y_{i+1}, p-2-y_{i+1}\right\}$

(c) $\mu_{i}\left(y_{i}\right) \in\left\{p-2-y_{i}, p-1-y_{i}\right\}$ implique $\mu_{i+1}\left(y_{i+1}\right) \in\left\{p-1-y_{i+1}, y_{i+1}-1\right\}$

ainsi que

$$
\begin{aligned}
\left(\mu_{0}\left(\lambda_{0}\left(r_{0}\right)\right), \cdots, \mu_{f-1}\left(\lambda_{f-1}\left(r_{f-1}\right)\right)\right) \otimes & \operatorname{det}^{e(\mu \circ \lambda)\left(r_{0}, \cdots, r_{f-1}\right)} \\
& =\left(\tau_{0}\left(r_{0}\right), \cdots, \tau_{f-1}\left(r_{f-1}\right)\right) \otimes \operatorname{det}^{e(\tau)\left(r_{0}, \cdots, r_{f-1}\right)},
\end{aligned}
$$

où $\left(\tau_{i}\left(x_{i}\right)\right)_{i} \in \mathcal{D}\left(x_{0}, \cdots, x_{f-1}\right)$ est le $f$-uplet correspondant au poids $\tau$ et où $\mu \circ \lambda:=$ $\left(\mu_{i}\left(\lambda_{i}\left(x_{i}\right)\right)\right)_{i}$. On en déduit que :

$$
\mu_{i}\left(\lambda_{i}\left(x_{i}\right)\right)=\tau_{i}\left(x_{i}\right), \quad \forall 0 \leq i \leq f-1 .
$$

En outre, le fait que $\tau$ soit indexé par $J^{\min }\left(=J^{\max }\right)$ implique grâce à $[8, \S 2]$ que l'on a

$$
\delta\left(\left\{i \mid \lambda_{i}\left(x_{i}\right) \in\left\{p-1-x_{i}, x_{i}+1, x_{i}+2\right\}\right\}\right)=\left\{i \mid \mu_{i}\left(y_{i}\right) \in\left\{p-1-y_{i}, p-2-y_{i}\right\}\right\} .
$$

D'après la condition (c) ci-dessus et la définition de $\delta$, ce qui équivaut à dire que :

$$
\lambda_{i}\left(x_{i}\right) \in\left\{p-1-x_{i}, x_{i}+1, x_{i}+2\right\} \Longleftrightarrow \mu_{i}\left(y_{i}\right) \in\left\{p-1-y_{i}, y_{i}-1\right\} .
$$

Rappelons que $J_{\tau}=\left\{i \mid \tau_{i}\left(x_{i}\right) \in\left\{x_{i}+1, p-3-x_{i}\right\}\right\}$ par définition. Sachant que $\mu_{i}\left(\lambda_{i}\left(x_{i}\right)\right)=$ $\tau_{i}\left(x_{i}\right)$, la condition $\tau_{i}\left(x_{i}\right)=x_{i}+1$ mène aux quatre possibilités suivantes pour $\lambda_{i}\left(x_{i}\right)$ et $\mu_{i}\left(y_{i}\right)$ :

$$
\begin{array}{lll}
\lambda_{i}\left(x_{i}\right)=x_{i}+1 & \text { et } & \mu_{i}\left(y_{i}\right)=y_{i} \\
\lambda_{i}\left(x_{i}\right)=p-2-x_{i} & \text { et } & \mu_{i}\left(y_{i}\right)=p-1-y_{i} \\
\lambda_{i}\left(x_{i}\right)=x_{i}+2 & \text { et } & \mu_{i}\left(y_{i}\right)=y_{i}-1 \\
\lambda_{i}\left(x_{i}\right)=p-3-x_{i} & \text { et } & \mu_{i}\left(y_{i}\right)=p-2-y_{i} .
\end{array}
$$


Or, seuls les deux derniers cas sont compatibles avec la condition (8), et l'on a donc $\lambda_{i}\left(x_{i}\right) \in$ $\left\{x_{i}+2, p-3-x_{i}\right\}$. De même, on vérifie que la condition $\tau_{i}\left(x_{i}\right)=p-3-x_{i}$ implique que $\lambda_{i}\left(x_{i}\right) \in\left\{x_{i}+2, p-3-x_{i}\right\}$, et on obtient donc que

$$
J_{\tau} \subseteq\left\{i \mid \lambda_{i}\left(x_{i}\right) \in\left\{p-3-x_{i}, x_{i}+2\right\}\right\} .
$$

Réciproquement, si $\lambda_{i}\left(x_{i}\right)=x_{i}+2$, on vérifie, de (8) et du fait que $\mu_{i}\left(\lambda_{i}\left(x_{i}\right)\right)=\tau_{i}\left(x_{i}\right)$, que seuls les deux cas suivants sont possibles pour $\tau_{i}\left(x_{i}\right)$ :

$$
\begin{array}{ll}
\tau_{i}\left(x_{i}\right)=x_{i}+1 & \left(\text { avec } \mu_{i}\left(y_{i}\right)=y_{i}-1\right) \\
\tau_{i}\left(x_{i}\right)=p-3-x_{i} & \left(\operatorname{avec} \mu_{i}\left(y_{i}\right)=p-1-y_{i}\right) .
\end{array}
$$

On en déduit que $i \in J_{\tau}$. On vérifie de la même façon que $\lambda_{i}\left(x_{i}\right)=p-3-x_{i}$ implique aussi $i \in J_{\tau}$, d'où le résultat.

Corollaire 2.2. Soit $\lambda \in \mathcal{P D}\left(x_{0}, \cdots, x_{f-1}\right)$ satisfaisant la condition (6) de telle sorte que $J^{\min }=J^{\max }=\delta\left(\left\{j \mid \lambda_{j}\left(x_{j}\right) \in\left\{p-1-x_{j}, x_{j}+1, x_{j}+2\right\}\right\}\right)$. Soit $\tau$ l'unique poids de $\mathcal{D}(\bar{\rho})$ tel que $\chi$ et $\chi^{s}$ apparaissent tous les deux sur $D_{\tau}(\bar{\rho})^{I_{1}\left(\mathcal{O}_{L}\right)}$. On a alors les équivalences suivantes :

$$
\begin{aligned}
& j \in \delta^{-1}\left(J^{\min }\right) \cap J_{\tau} \Longleftrightarrow \lambda_{j}\left(x_{j}\right)=x_{j}+2 \\
& j \in \delta^{-1}\left(J^{\min }\right) \backslash J_{\tau} \Longleftrightarrow \lambda_{j}\left(x_{j}\right) \in\left\{p-1-x_{j}, x_{j}+1\right\} \\
& j \in J_{\tau} \backslash \delta^{-1}\left(J^{\min }\right) \Longleftrightarrow \lambda_{j}\left(x_{j}\right)=p-3-x_{j} \\
& j \notin J_{\tau} \cup \delta^{-1}\left(J^{\mathrm{min}}\right) \Longleftrightarrow \lambda_{j}\left(x_{j}\right) \in\left\{p-2-x_{j}, x_{j}\right\} .
\end{aligned}
$$

Démonstration. Conséquence immédiate de la proposition 2.1 .

Pour $\lambda \in \mathcal{P D}\left(x_{0}, \cdots, x_{f-1}\right)$ et $\tau$ comme dans le corollaire 2.2 , on pose :

$$
I(\lambda):=\delta^{-1}\left(J^{\min }\right) \backslash J_{\tau} \coprod J_{\tau} \backslash \delta^{-1}\left(J^{\min }\right) .
$$

Autrement dit, on a $I(\lambda)=\left\{j \mid \lambda_{j}\left(x_{j}\right) \in\left\{x_{j}+1, p-1-x_{j}, p-3-x_{j}\right\}\right\}$. On obtient alors immédiatement l'énoncé suivant, conséquence des définitions de $\mathcal{P D}\left(x_{0}, \cdots, x_{f-1}\right)$ et de $I(\lambda)$.

Corollaire 2.3. Soit $\lambda \in \mathcal{P D}\left(x_{0}, \cdots, x_{f-1}\right)$ vérifiant la condition (6). On a les équivalences suivantes :

$$
\begin{array}{cc}
\lambda_{j}\left(x_{j}\right) \in\left\{p-1-x_{j}, p-2-x_{j}, p-3-x_{j}\right\} & \Longleftrightarrow j+1 \in I(\lambda) \\
\lambda_{j}\left(x_{j}\right) \in\left\{x_{j}, x_{j}+1, x_{j}+2\right\} & \Longleftrightarrow j+1 \notin I(\lambda) .
\end{array}
$$

Le corollaire suivant est un cas particulier de la proposition 2.1.

Corollaire 2.4. Supposons que la représentation $\bar{\rho}$ soit scindée. Soit $\lambda \in \mathcal{P D}\left(x_{0}, \cdots, x_{f-1}\right)$ et soit $\chi:=\tau(\lambda)^{I_{1}\left(\mathcal{O}_{L}\right)}$ le caractère de $I\left(\mathcal{O}_{L}\right)$ correspondant à $\lambda$. Les caractères $\chi$ et $\chi^{s}$ apparaissent sur le même facteur $D_{\tau}(\bar{\rho})^{I_{1}\left(\mathcal{O}_{L}\right)}$ avec $\tau \in \mathcal{D}(\bar{\rho})$ si et seulement si l'une des conditions suivantes est vérifiée :

(i) ou bien $\lambda_{j}\left(x_{j}\right) \in\left\{x_{j}, x_{j}+2\right\}$ pour tout $j$, auquel cas $J_{\tau}=\left\{j \mid \lambda_{j}\left(x_{j}\right)=x_{j}+2\right\}$

(ii) ou bien $\lambda_{j}\left(x_{j}\right) \in\left\{p-1-x_{j}, p-3-x_{j}\right\}$ pour tout $j$, auquel cas $J_{\tau}=\left\{j \mid \lambda_{j}\left(x_{j}\right)=\right.$ $\left.p-3-x_{j}\right\}$. 
Démonstration. Supposons que $\chi$ et $\chi^{S}$ apparaissent tous les deux sur $D_{\tau}(\bar{\rho})^{I_{1}\left(\mathcal{O}_{L}\right)}$. Comme $\bar{\rho}$ est scindée, on a $J_{\bar{\rho}}=\mathcal{S}$ d'après (2), et la condition (6) se traduit alors par :

$$
\forall 0 \leq j \leq f-1, \quad \lambda_{j}\left(x_{j}\right) \in\left\{x_{j}, x_{j}+2, p-1-x_{j}, p-3-x_{j}\right\} .
$$

On 'en déduit facilement l'énoncé voulu grâce à la définition de $\mathcal{P D}\left(x_{0}, \cdots, x_{f-1}\right)$ et à la proposition 2.1 .

Corollaire 2.5. Avec les notations précédentes, le nombre de paires (non ordonnées) $\left\{\chi, \chi^{s}\right\}$ telles que $\chi$ et $\chi^{s}$ apparaissent tous les deux sur le même facteur $D_{\tau}(\bar{\rho})^{I_{1}\left(\mathcal{O}_{L}\right)}$ avec $\tau \in \mathcal{D}(\bar{\rho})$ est $2^{f-1}$ (resp. $2^{f}$ ) si $\bar{\rho}$ est non scindée (resp. scindée).

Démonstration. C'est une conséquence de [5, Prop. 4.4]. En effet, loc. cit. entraîne que pour chaque sous-ensemble $J^{\text {min }}=J^{\text {max }} \subseteq \mathcal{S}$, il existe un unique $\chi$ si $\bar{\rho}$ est non scindée (resp. exactement deux $\chi$ si $\bar{\rho}$ est scindée) tel(s) que $\chi$ et $\chi^{s}$ apparaissent tous les deux sur le facteur $D_{\tau}(\bar{\rho})^{I_{1}\left(\mathcal{O}_{L}\right)}$, où $\tau \in \mathcal{D}(\bar{\rho})$ est le poids sous-quotient de $\bar{\sigma}\left(\chi^{s}\right)$ indexé par $J^{\text {min }}$. Le nombre de tels $\chi$ obtenus est donc $2^{f}$ (resp. $2^{f+1}$ ) dans le cas non scindé (resp. scindé) et le résultat pour les paires non ordonnées s'en déduit en divisant par 2.

Notons que dans tous les deux cas, on peut redémontrer [5, Prop. 4.4] en utilisant respectivement le corollaire 2.2 et le corollaire 2.4 .

Une raison pour laquelle on ne considère que les $\lambda \in \mathcal{P D}\left(x_{0}, \cdots, x_{f-1}\right)$ vérifiant (6) est donnée par la proposition suivante.

Proposition 2.6. Soit $\lambda$ vérifiant (6) comme précédemment et $\chi$ le caractère correspondant $\grave{a} \lambda$. Soit $\tau \in \mathcal{D}(\bar{\rho})$ comme dans le corollaire 2.2 et soit $s_{i}:=\tau_{i}\left(r_{i}\right)$ pour tout $0 \leq i \leq f-1$, de sorte que $\tau=\left(s_{0}, \cdots, s_{f-1}\right) \otimes \operatorname{det}^{e(\tau)\left(r_{0}, \cdots, r_{f-1}\right)}$. Soit $\pi$ une représentation lisse de $\mathrm{GL}_{2}(L)$ sur $k_{E}$ vérifiant les propriétés suivantes:

(i) $p \in L^{\times}$agit trivialement sur $\pi$

(ii) $\pi$ contient $D(\bar{\rho})$

(iii) $D(\bar{\rho})^{I_{1}\left(\mathcal{O}_{L}\right)}$ est stable sous l'action de $\left(\begin{array}{ll}0 & 1 \\ p & 0\end{array}\right)$ sur $\pi$.

Alors il existe (à multiplication par un scalaire non nul près) un unique vecteur $v(\lambda) \in$ $D_{\tau}(\bar{\rho})^{I_{1}\left(\mathcal{O}_{L}\right)} \subset \pi^{I_{1}\left(\mathcal{O}_{L}\right)}$ non nul sur lequel $I\left(\mathcal{O}_{L}\right)$ agit par $\chi^{s}$ et un unique élément $x(\lambda) \in k_{E}^{\times}$ tel que l'on ait l'égalité suivante dans $\pi$ :

$$
\begin{aligned}
\sum_{t \in \mathbb{F}_{q}}\left(\prod_{j \notin J^{\min }} \sigma_{0}(t)^{p^{j}\left(p-1-s_{j}\right)}\right)\left(\begin{array}{cc}
{[t]} & 1 \\
1 & 0
\end{array}\right)\left(\begin{array}{ll}
0 & 1 \\
p & 0
\end{array}\right) v(\lambda) \\
\quad=x(\lambda) \sum_{t \in \mathbb{F}_{q}}\left(\prod_{j \in J^{\min }} \sigma_{0}(t)^{p^{j}\left(p-1-s_{j}\right)}\right)\left(\begin{array}{cc}
t] & 1 \\
1 & 0
\end{array}\right) v(\lambda) .
\end{aligned}
$$

Démonstration. C'est une conséquence de [8, Lem. 2.7] et de la réciprocité de Frobenius. D'abord, l'existence et l'unicité (à un scalaire près) de $v(\lambda)$ découlent du fait que le sousespace propre de caractère $\chi^{s}$ de $D_{\tau}(\bar{\rho})^{I_{1}\left(\mathcal{O}_{L}\right)}$ est de dimension 1 sur $k_{E}$. Comme le poids $\tau$ est indexé par $J^{\min }\left(=J^{\max }\right)$ dans $\bar{\sigma}\left(\chi^{s}\right)$, on sait d'après [8, Lem. 2.7] que le vecteur :

$$
\sum_{t \in \mathbb{F}_{q}}\left(\prod_{j \in J^{\min }} \sigma_{0}(t)^{p^{j}\left(p-1-s_{j}\right)}\right)\left(\begin{array}{cc}
{[t]} & 1 \\
1 & 0
\end{array}\right) v(\lambda)
$$


est un générateur de $\tau^{I_{1}\left(\mathcal{O}_{L}\right)}$ dans $D_{\tau}(\bar{\rho})$. D'autre part, l'hypothèse (iii) implique que le vecteur $\left(\begin{array}{ll}0 & 1 \\ p & 0\end{array}\right) v(\lambda)$, sur lequel $I\left(\mathcal{O}_{L}\right)$ agit par $\chi$, appartient à $D_{\tau}(\bar{\rho})^{I_{1}\left(\mathcal{O}_{L}\right)}$. Le même argument appliqué à $\bar{\sigma}(\chi)$ et à $\left(\begin{array}{ll}0 & 1 \\ p & 0\end{array}\right) v(\lambda)$ montre que le vecteur de gauche de (10) est aussi un générateur de $\tau^{I_{1}\left(\mathcal{O}_{L}\right)}$ dans $D_{\tau}(\bar{\rho}), \tau$ étant cette fois indexé par $\mathcal{S} \backslash J^{\min }$ dans $\bar{\sigma}(\chi)$, ce qui prouve le corollaire puisque $\operatorname{dim}_{k_{E}} \tau^{I_{1}\left(\mathcal{O}_{L}\right)}=1$.

Remarque 2.7. Reprenons les notations de la proposition 2.1 et supposons que $\chi$ est le caractère " $\eta(J) \otimes \eta^{\prime}(J)$ " où $\eta(J)$ et $\eta^{\prime}(J)$ sont définis par [6, (3)] pour une partie $J$ de $\{0, \cdots, f-1\}$, ce dernier ensemble étant identifié à $\left\{\sigma: \mathbb{F}_{q} \hookrightarrow k_{E}\right\}$ via $j \mapsto \sigma_{0} \circ \varphi^{-j}$.

(i) On retrouve [6, Lem.2.1.4] puisque $J_{\tau}=\emptyset$ d'après [6, Lem.2.1.3].

(ii) Notre $J^{\mathrm{min}}$ correspondant à $\chi$ est égal à $\mathcal{S} \backslash J$, de sorte que la proposition 2.6 est compatible avec [6, Prop.2.6.1]. En effet, avec les notations de [6], on a $\mathcal{S} \backslash J=\left\{j \mid c_{\sigma_{0} \circ \varphi^{j}} \in\right.$ $\left.\left\{p-1-r_{j}, p-2-r_{j}\right\}\right\}$ d'après [6, Lem.2.1.3].

\section{Modules fortement divisibles et leurs réductions}

On calcule les modules de Fontaine-Laffaille de représentations réductibles de Gal $\left(\overline{\mathbb{Q}}_{p} / L\right)$ sur $k_{E}$ provenant de certains modules fortement divisibles avec donnée de descente modérément ramifiée.

On pose $e:=p^{f}-1$ et l'on définit $S$ comme le complété $p$-adique de l'enveloppe aux puissances divisées de $\mathcal{O}_{E}[u]$ par rapport à l'idéal $\left(u^{e}+p\right) \mathcal{O}_{E}[u]$ compatibles avec les puissances divisées sur l'idéal $p \mathcal{O}_{E}[u]$. On note $\mathrm{Fil}^{p} S$ le complété $p$-adique de l'idéal engendré par $\frac{\left(u^{e}+p\right)^{i}}{i !}$ pour $i \geq p$. Soient $\eta, \eta^{\prime}: \mathbb{F}_{q}^{\times} \rightarrow \mathcal{O}_{E}^{\times}$deux caractères distincts. Notons $c=\sum_{i=0}^{f-1} p^{i} c_{i} \in\{1, \cdots, q-2\}$ l'unique entier tel que $\eta \eta^{\prime-1}=\left[\sigma_{0}\right]^{c}$ (où $[\cdot]: k_{E}^{\times} \rightarrow \mathcal{O}_{E}^{\times}$ désigne le représentant multiplicatif) et posons pour $j \in \mathcal{S}=\{0, \cdots, f-1\}$

$$
c^{(j)}:=\sum_{i=0}^{j-1} c_{f-(j-i)} p^{i}+\sum_{i=j}^{f-1} c_{i-j} p^{i} \in\{1, \cdots, q-2\} .
$$

On renvoie à $[5, \S 5]$ ou $[6, \S 2]$ pour la définition d'un $\mathcal{O}_{E^{-}}$module fortement divisible de type $\eta \otimes \eta^{\prime}$ que l'on utilisera. Dans cet article, nous n'aurons besoin de considérer que les modules qui ont la forme définie (à l'exception de (iv) ci-après) au début de [6, §2.2]. Concrètement, les $\mathcal{O}_{E}$-modules fortement divisibles qui nous intéressent dans cet article ont la forme générale suivante :

(i) $\mathcal{M}=\mathcal{M}^{\sigma_{0}} \times \cdots \times \mathcal{M}^{\sigma_{0} \circ \varphi^{-(f-1)}}$ avec $\mathcal{M}^{\sigma_{0} \circ \varphi^{-j}}=S e_{\eta}^{\sigma_{0} \circ \varphi^{-j}} \oplus S e_{\eta^{\prime}}^{\sigma_{0} \circ \varphi^{-j}}$

(ii) pour tout $j \in \mathcal{S}, \operatorname{Gal}(L[\sqrt[e]{-p}] / L)$ agit $\operatorname{sur} e_{\eta}^{\sigma_{0} \circ \varphi^{-j}}\left(\right.$ resp. $\left.e_{\eta^{\prime}}^{\sigma_{0} \circ \varphi^{-j}}\right)$ par $\eta\left(\right.$ resp. $\left.\eta^{\prime}\right)$

(iii) pour tout $j \in \mathcal{S}$, l'application $\varphi_{1}: \operatorname{Fil}^{1} \mathcal{M}^{\sigma_{0} \circ \varphi^{-j}} \rightarrow \mathcal{M}^{\sigma_{0} \circ \varphi^{-(j+1)}}$ vérifie l'une des deux configurations suivantes (où l'on remplace $\sigma_{0} \circ \varphi^{-j}$ par $j$ pour alléger l'écriture) :

$$
\begin{cases}\operatorname{Fil}^{1} \mathcal{M}^{j} & =\left(S\left(e_{\eta}^{j}+a_{j} u^{c^{(j)}} e_{\eta^{\prime}}^{j}\right) \oplus S\left(u^{e}+p\right) e_{\eta^{\prime}}^{j}\right)+\mathrm{Fil}^{p} S \cdot \mathcal{M}^{j} \\ \varphi_{1}\left(e_{\eta}^{j}+a_{j} u^{c^{(j)}} e_{\eta^{\prime}}^{j}\right) & =e_{\eta}^{j+1} \\ \varphi_{1}\left(\left(u^{e}+p\right) e_{\eta^{\prime}}^{j}\right) & =e_{\eta^{\prime}}^{j+1}\end{cases}
$$


$\mathrm{Ou}$

$$
\begin{cases}\operatorname{Fil}^{1} \mathcal{M}^{j} & =\left(S\left(u^{e}+p\right) e_{\eta}^{j} \oplus S\left(e_{\eta^{\prime}}^{j}+a_{j} u^{e-c^{(j)}} e_{\eta}^{j}\right)\right)+\mathrm{Fil}^{p} S \cdot \mathcal{M}^{j} \\ \varphi_{1}\left(\left(u^{e}+p\right) e_{\eta}^{j}\right) & =e_{\eta}^{j+1} \\ \varphi_{1}\left(e_{\eta^{\prime}}^{j}+a_{j} u^{e-c^{(j)}} e_{\eta}^{j}\right) & =e_{\eta^{\prime}}^{j+1}\end{cases}
$$

avec $a_{j}=a_{\sigma_{0} \circ \varphi^{-j}} \in \mathcal{O}_{E}$ et avec $\alpha e_{\eta}^{0}$ et $\alpha^{\prime} e_{\eta^{\prime}}^{0}\left(\alpha, \alpha^{\prime} \in \mathcal{O}_{E}^{\times}\right)$au lieu de $e_{\eta}^{j+1}$ et $e_{\eta^{\prime}}^{j+1}$ dans l'image de $\varphi_{1}$ lorsque $j=f-1$. On note $I_{\eta}$ (resp. $I_{\eta^{\prime}}$ ) le sous-ensemble de $\mathcal{S}$ formé des $j$ tels que $\mathcal{M}^{j}$ soit comme en (11) (resp. comme en (12)) et $I_{\eta}^{\times}$(resp. $I_{\eta^{\prime}}^{\times}$) le sous-ensemble de $I_{\eta}$ (resp. $I_{\eta}^{\prime}$ ) formé des $j$ tels que $a_{j} \in \mathcal{O}_{E}^{\times}$. On pose enfin $I_{\eta}^{0}=I_{\eta} \backslash I_{\eta}^{\times}, I_{\eta^{\prime}}^{0}=I_{\eta^{\prime}} \backslash I_{\eta^{\prime}}^{\times}$, et l'on ajoute la condition suivante

(iv) On demande que $\overline{\alpha \alpha}^{\prime}=1$.

Remarque 3.1. Dans [5], un autre type noté "cas II" est considéré. Il n'apparîtra pas dans notre étude parce que la condition (6) implique $J^{\min }=J^{\max }$, de sorte que l'on a ici $\mathrm{II}=J^{\max } \backslash J^{\min }=\emptyset(c f .[5,(26)])$.

On note $\bar{S}=S \otimes_{\mathcal{O}_{E}} k_{E}$ et $\overline{\mathcal{M}}=\mathcal{M} \otimes_{\mathcal{O}_{E}} k_{E}$ pour $\mathcal{M}$ un $\mathcal{O}_{E}$-module fortement divisible de type $\eta \otimes \eta^{\prime}$ comme ci-dessus. On pose aussi

$$
\bar{\rho}_{\mathcal{M}}=\operatorname{Hom}_{\mathrm{Fil}^{1}, \varphi_{1}}\left(\overline{\mathcal{M}}, \widehat{A}_{\text {cris }} \otimes_{\mathbb{Z}_{p}} \mathbb{F}_{p}\right)^{\vee}(1)
$$

et l'on renvoie à $[14, \S 3]$ pour plus de détails concernant le membre de droite. D'après $[5$, Thm.8.1], $\bar{\rho}_{\mathcal{M}}$ est toujours réductible et elle est scindée si et seulement si $I_{\eta}^{\times} \amalg I_{\eta^{\prime}}^{\times}=\emptyset$. De manière équivalente, $\overline{\mathcal{M}}$ contient toujours un sous- $\varphi_{1}$-module filtré (pour la filtration induite) de rang 1 qui est facteur direct en tant que $\bar{S}$-module, stable par $\operatorname{Gal}\left(\overline{\mathbb{Q}}_{p} / L\right)$, et ce sous-module est unique si et seulement si $\bar{\rho}$ est non scindée. Par [5, Lem. 6.1], un tel sous-module est de la forme $\prod_{j=0}^{f-1} \bar{S} e^{j}$, chaque $e^{j}$ étant égal à $\bar{e}_{\eta}^{j}+\beta_{j} u^{p c^{(j-1)}} \bar{e}_{\eta^{\prime}}^{j}$ ou à $\bar{e}_{\eta^{\prime}}^{j}+\beta_{j} u^{p\left(e-c^{(j-1)}\right)} \bar{e}_{\eta}^{j}$ avec $\beta_{j} \in k_{E}$, où $\bar{e}_{\eta}^{j}$ (resp. $\bar{e}_{\eta^{\prime}}^{j}$ ) désigne l'image de $e_{\eta}^{j}$ (resp. $e_{\eta^{\prime}}^{j}$ ) dans $\overline{\mathcal{M}}$. On peut en outre lui associer une suite de caractères $\left\{\eta_{j}\right\}_{j \in \mathcal{S}}$ en posant $\eta_{j}=\eta$ lorsque $e_{j}$ vérifie le premier cas, et $\eta_{j}=\eta^{\prime}$ sinon. D'après [5, Lem.6.3(iii)], on a la récurrence suivante :

$$
\left\{\begin{array}{l}
\eta_{j+1}=\eta_{j} \quad \text { si } \quad j \in I_{\eta}^{0} \amalg I_{\eta^{\prime}}^{0} \\
\eta_{j+1}=\eta^{\prime} \quad \text { si } j \in I_{\eta}^{\times} \\
\eta_{j+1}=\eta \quad \text { si } j \in I_{\eta^{\prime}}^{\times}
\end{array}\right.
$$

Si $\bar{\rho}_{\mathcal{M}}$ est non scindée, la suite $\left\{\eta_{j}\right\}_{j}$ est uniquement déterminée par $\mathcal{M}$ puisque $I_{\eta}^{\times} \coprod I_{\eta^{\prime}}^{\times} \neq \emptyset$. Si $\bar{\rho}_{\mathcal{M}}$ est scindée, alors $\overline{\mathcal{M}}$ est somme directe de deux sous- $\varphi_{1}$-modules filtrés de rang 1 respectivement associés aux suites constantes $\left\{\eta_{j}=\eta\right\}_{j}$ et $\left\{\eta_{j}=\eta^{\prime}\right\}_{j}$.

La proposition suivante est une généralisation de [6, Prop.2.2.4].

Proposition 3.2. Soient $\mathcal{M}$ un $\mathcal{O}_{E}$-module fortement divisible de type $\eta \otimes \eta^{\prime}$ et $\left\{\eta_{j}\right\}_{j}$ une suite vérifiant la récurrence (14) ci-dessus. On rappelle que les entiers $c_{j}$ sont définis par la relation $\eta \eta^{-1}=\left[\sigma_{0}\right]^{\sum_{j=0}^{f-1} p^{i} c_{j}}$. On a alors $\operatorname{det} \bar{\rho}_{\mathcal{M}}=\overline{\eta \eta}^{\prime} \omega$ et

$$
\bar{\rho}_{\mathcal{M}} \cong\left(\begin{array}{cc}
\operatorname{nr}(A) \omega_{\sigma_{0}}^{\sum_{j=0}^{f-1} p^{j}\left(r_{j}^{\prime}+1\right)} & * \\
0 & \operatorname{nr}\left(A^{-1}\right)
\end{array}\right) \otimes \theta,
$$


où $\theta$ est un caractère convenable uniquement déterminé, où $r_{j}^{\prime} \in\{0, \cdots, p-3\}$ vérifie

$$
\left\{\begin{array}{ccccc}
r_{f-j}^{\prime}=c_{f-j}-2 & \text { si } & j \in I_{\eta}^{0} & \text { et } & \eta_{j}=\eta \\
r_{f-j}^{\prime}=p-1-c_{f-j} & \text { si } & j \in I_{\eta} & \text { et } & \eta_{j}=\eta^{\prime} \\
r_{f-j}^{\prime}=c_{f-j} & \text { si } j \in I_{\eta^{\prime}} & \text { et } & \eta_{j}=\eta \\
r_{f-j}^{\prime}=p-3-c_{f-j} & \text { si } j \in I_{\eta^{\prime}}^{0} & \text { et } & \eta_{j}=\eta^{\prime} \\
r_{f-j}^{\prime}=c_{f-j}-1 & \text { si } & j \in I_{\eta}^{\times} & \text {et } & \eta_{j}=\eta \\
r_{f-j}^{\prime}=p-2-c_{f-j} & \text { si } & j \in I_{\eta^{\prime}}^{\times} & \text {et } & \eta_{j}=\eta^{\prime},
\end{array}\right.
$$

et où

$$
A=\left\{\begin{array}{cccc}
\bar{\alpha} \prod_{\left\{j \mid \eta_{j} \neq \eta_{j+1}\right\}} \bar{a}_{i} & \text { si } & \eta_{0}=\eta \\
\bar{\alpha}^{-1} \prod_{\left\{j \mid \eta_{j} \neq \eta_{j+1}\right\}} \bar{a}_{i} & \text { si } & \eta_{0}=\eta^{\prime} .
\end{array}\right.
$$

Démonstration. L'assertion sur $\operatorname{det} \bar{\rho}_{\mathcal{M}}$ se vérifie par un calcul direct.

Montrons maintenant (15) et (16). On explicite tout d'abord un sous- $\varphi_{1}$-module filtré de rang 1 qui soit facteur direct et stable par toutes les opérations. On pose, pour $j \in$ $\{0, \cdots, f-1\}$ :

$$
\begin{aligned}
& e^{j+1}=\bar{e}_{\eta}^{j+1} \quad \text { si } j \in I_{\eta}^{0} \text { et } \eta_{j}=\eta \\
& e^{j+1}=\bar{e}_{\eta^{\prime}}^{j+1} \quad \text { si } j \in I_{\eta}^{0} \text { et } \eta_{j}=\eta^{\prime} \\
& e^{j+1}=\bar{e}_{\eta}^{j+1} \quad \text { si } j \in I_{\eta^{\prime}}^{0} \text { et } \eta_{j}=\eta \\
& e^{j+1}=\bar{e}_{\eta^{\prime}}^{j+1} \quad \text { si } j \in I_{\eta^{\prime}}^{0} \text { et } \eta_{j}=\eta^{\prime} \\
& e^{j+1}=\bar{e}_{\eta^{\prime}}^{j+1}-\bar{a}_{j}^{-1} u^{p\left(e-c^{(j)}\right)} \bar{e}_{\eta}^{j+1} \quad \text { si } \quad j \in I_{\eta}^{\times} \quad \text { et } \quad \eta_{j}=\eta \\
& e^{j+1}=\quad \bar{e}_{\eta^{\prime}}^{j+1} \quad \text { si } j \in I_{\eta}^{\times} \text {et } \eta_{j}=\eta^{\prime} \\
& e^{j+1}=\bar{e}_{\eta}^{j+1} \quad \text { si } j \in I_{\eta^{\prime}}^{\times} \text {et } \eta_{j}=\eta \\
& e^{j+1}=\bar{e}_{\eta}^{j+1}-\bar{a}_{j}^{-1} u^{p c^{(j)}} \bar{e}_{\eta^{\prime}}^{j+1} \quad \text { si } \quad j \in I_{\eta^{\prime}}^{\times} \quad \text { et } \quad \eta_{j}=\eta^{\prime}
\end{aligned}
$$

en remplaçant $\bar{a}_{j}^{-1}$ par $\bar{a}_{f-1}^{-1} \bar{\alpha}^{-2}$ (resp. $\left.\bar{a}_{f-1}^{-1} \bar{\alpha}^{2}\right)$ si $j=f-1$ et $\eta_{0}=\eta$ (resp. $\eta_{0}=\eta^{\prime}$ ). Nous allons vérifier que le sous-module $\overline{\mathcal{N}}:=\bar{S} e^{0} \times \cdots \times \bar{S} e^{f-1}$ de $\overline{\mathcal{M}}$, muni de la filtration induite, est stable par toutes les opérations et que si $h_{j} \in\{0, \cdots, e\}$ est le plus petit entier tel que $u^{h_{j}} e^{j} \in \mathrm{Fil}^{1} \overline{\mathcal{N}}$, alors :

$$
\begin{array}{llcllll}
h_{j}=0 & \text { et } & \varphi_{1}\left(e^{j}\right)=e^{j+1} & \text { si } & j \in I_{\eta}^{0} & \text { et } & \eta_{j}=\eta \\
h_{j}=e & \text { et } & \varphi_{1}\left(e^{j}\right)=e^{j+1} & \text { si } & j \in I_{\eta}^{0} & \text { et } & \eta_{j}=\eta^{\prime} \\
h_{j}=e & \text { et } & \varphi_{1}\left(e^{j}\right)=e^{j+1} & \text { si } & j \in I_{\eta^{\prime}}^{0} & \text { et } & \eta_{j}=\eta \\
h_{j}=0 & \text { et } & \varphi_{1}\left(e^{j}\right)=e^{j+1} & \text { si } & j \in I_{\eta^{\prime}}^{0} & \text { et } & \eta_{j}=\eta^{\prime} \\
h_{j}=e-c^{(j)} & \text { et } & \varphi_{1}\left(e^{j}\right)=-\bar{a}_{j} e^{j+1} & \text { si } & j \in I_{\eta}^{\times} & \text {et } & \eta_{j}=\eta \\
h_{j}=e & \text { et } & \varphi_{1}\left(e^{j}\right)=e^{j+1} & \text { si } & j \in I_{\eta}^{\times} & \text {et } & \eta_{j}=\eta^{\prime} \\
h_{j}=e & \text { et } & \varphi_{1}\left(e^{j}\right)=e^{j+1} & \text { si } & j \in I_{\eta^{\prime}}^{\times} & \text {et } & \eta_{j}=\eta \\
h_{j}=c^{(j)} & \text { et } & \varphi_{1}\left(e^{j}\right)=-\bar{a}_{j} e^{j+1} & \text { si } & j \in I_{\eta^{\prime}}^{\times} & \text {et } & \eta_{j}=\eta^{\prime}
\end{array}
$$

en multipliant le terme $e^{j+1}$ ou $-\bar{a}_{j} e^{j+1}$ par $\bar{\alpha}\left(\right.$ resp. $\left.\bar{\alpha}^{-1}\right)$ si $j=f-1$ et $\eta_{0}=\eta$ (resp. $\left.\eta_{0}=\eta^{\prime}\right)$.

Il'est clair que $\overline{\mathcal{N}}$ est stable par l'action de $\operatorname{Gal}(L[\sqrt[e]{-p}] / L)$. Vérifions maintenant que l'image de Fil ${ }^{1} \overline{\mathcal{N}}$ par $\varphi_{1}$ est contenue dans $\overline{\mathcal{N}}$ et engendre $\overline{\mathcal{N}}$ sur $\bar{S}$, i.e. $\varphi_{1}\left(u^{h_{j}} e^{j}\right) \in \bar{S}^{\times} e^{j+1}$ pour tout $j$. Nous traitons le cas où $\eta_{j}=\eta$ et $j \neq f-1$, les autres cas étant laissés au 
lecteur. Supposons donc $\eta_{j}=\eta$ et $j \neq f-1$. Par définition et d'après la récurrence (14), on dispose des possibilités suivantes pour $\eta_{j-1}$ et $e^{j}$ :

$$
\begin{aligned}
& e^{j}=\quad \bar{e}_{\eta}^{j} \quad \text { si } \quad j-1 \in I_{\eta}^{0} \amalg I_{\eta^{\prime}} \quad \text { et } \quad \eta_{j-1}=\eta \\
& e^{j}=\bar{e}_{\eta}^{j}-\bar{a}_{j-1}^{-1} u^{p c^{(j-1)}} \bar{e}_{\eta^{\prime}}^{j} \quad \text { si } \quad j-1 \in I_{\eta^{\prime}}^{\times} \quad \text { et } \quad \eta_{j-1}=\eta^{\prime} \text {. }
\end{aligned}
$$

La vérification se fait au cas par cas comme suit. Rappelons que, d'après [5, Lem. 6.3(ii)], on a toujours

$$
\varphi_{1}\left(u^{h_{j}} e^{j}\right)=\varphi_{1}\left(u^{h_{j}} \bar{e}_{\eta}^{j}\right)
$$

(a) Supposons que $j \in I_{\eta}^{0}$ (et $\eta_{j}=\eta$ ). Puisque val $\left(a_{j}\right)>0$, on sait par (11) que :

$$
\operatorname{Fil}^{1} \overline{\mathcal{M}}^{j}=\bar{S} \bar{e}_{\eta}^{j} \oplus \bar{S} u^{e} \bar{e}_{\eta^{\prime}}^{j}, \quad \varphi_{1}\left(\bar{e}_{\eta}^{j}\right)=\bar{e}_{\eta}^{j+1}, \quad \varphi_{1}\left(u^{e} \bar{e}_{\eta^{\prime}}^{j}\right)=\bar{e}_{\eta^{\prime}}^{j+1} .
$$

Dans le premier cas de (18), on a clairement $h_{j}=0$, puis l'on déduit de (19) que $\varphi_{1}\left(e^{j}\right)=\varphi_{1}\left(\bar{e}_{\eta}^{j}\right)=\bar{e}_{\eta}^{j+1}=e^{j+1}$ comme annoncé. Dans le deuxième cas de (18), en notant que $p c^{(j-1)}>e$ puisque $c_{f-j}>1$ grâce à [5, Lem.6.3], on a $e^{j} \in \mathrm{Fil}^{1} \overline{\mathcal{M}}^{j}$ donc on a encore $h_{j}=0$ puis $\varphi_{1}\left(e^{j}\right)=\varphi_{1}\left(\bar{e}_{\eta}^{j}\right)=e^{j+1}$ par (19).

(b) Supposons que $j \in I_{\eta^{\prime}}^{0}$ (et $\eta_{j}=\eta$ ). Comme val $\left(a_{j}\right)>0$, on déduit de (12) que :

$$
\operatorname{Fil}^{1} \overline{\mathcal{M}}^{j}=\bar{S} u^{e} \bar{e}_{\eta}^{j} \oplus \bar{S} \bar{e}_{\eta^{\prime}}^{j}, \quad \varphi_{1}\left(u^{e} \bar{e}_{\eta}^{j}\right)=\bar{e}_{\eta}^{j+1}, \quad \varphi_{1}\left(\bar{e}_{\eta^{\prime}}^{j}\right)=\bar{e}_{\eta^{\prime}}^{j+1}
$$

Dans le premier cas de (18), on a $h_{j}=e$ et $\varphi_{1}\left(u^{e} e^{j}\right)=\varphi_{1}\left(u^{e} \bar{e}_{\eta}^{j}\right)=\bar{e}_{\eta}^{j+1}=e^{j+1}$. Dans le deuxième cas de (18), on a encore $h_{j}=e$ et $\varphi_{1}\left(u^{e} e^{j}\right)=\varphi_{1}\left(u^{e} \bar{e}_{\eta}^{j}\right)=e^{j+1} \operatorname{par}(19)$.

(c) Supposons que $j \in I_{\eta}^{\times}$(et $\eta_{j}=\eta$ ). On obtient alors de (11) que :

$$
\operatorname{Fil}^{1} \overline{\mathcal{M}}^{j}=\bar{S}\left(\bar{e}_{\eta}^{j}+\bar{a}_{j} u^{c^{(j)}} \bar{e}_{\eta^{\prime}}^{j}\right) \oplus \bar{S} u^{e} \bar{e}_{\eta^{\prime}}^{j}, \quad \varphi_{1}\left(\bar{e}_{\eta}^{j}+\bar{a}_{j} u^{c^{(j)}} \bar{e}_{\eta^{\prime}}^{j}\right)=\bar{e}_{\eta}^{j+1}, \quad \varphi_{1}\left(u^{e} \bar{e}_{\eta^{\prime}}^{j}\right)=\bar{e}_{\eta^{\prime}}^{j+1} .
$$

Dans le premier cas de (18), on a

$$
u^{e-c^{(j)}} e^{j}=u^{e-c^{(j)}} \bar{e}_{\eta}^{j}=u^{e-c^{(j)}}\left(\bar{e}_{\eta}^{j}+\bar{a}_{j} u^{c^{(j)}} \bar{e}_{\eta^{\prime}}^{j}\right)-\bar{a}_{j} u^{e} \bar{e}_{\eta^{\prime}}^{j} \in \mathrm{Fil}^{1} \overline{\mathcal{M}}^{j}
$$

et on voit facilement que $e-c^{(j)}$ est le plus petit entier vérifiant cette condition, i.e. $h_{j}=e-c^{(j)}$. On en déduit le résultat voulu dans ce cas :

$$
\varphi_{1}\left(u^{e-c^{(j)}} e^{j}\right)=u^{p\left(e-c^{(j)}\right)} \bar{e}_{\eta}^{j+1}-\bar{a}_{j} \bar{e}_{\eta^{\prime}}^{j+1}=-\bar{a}_{j}\left(\bar{e}_{\eta^{\prime}}^{j+1}-\bar{a}_{j}^{-1} u^{p\left(e-c^{(j)}\right)} \bar{e}_{\eta}^{j+1}\right)
$$

qui nous donne exactement $-\bar{a}_{j} e^{j+1}$ par définition de $e^{j+1}$. Dans le deuxième cas de (18), on a

$$
u^{e-c^{(j)}} e^{j}=u^{e-c^{(j)}}\left(\bar{e}_{\eta}^{j}-\bar{a}_{j-1}^{-1} u^{p c^{(j-1)}} \bar{e}_{\eta^{\prime}}^{j}\right)=u^{e-c^{(j)}} \bar{e}_{\eta}^{j}-\bar{a}_{j-1}^{-1} u^{e-c^{(j)}+p c^{(j-1)}} \bar{e}_{\eta^{\prime}}^{j},
$$

qui appartient à Fil ${ }^{1} \overline{\mathcal{M}}^{j}$ puisque $u^{e-c^{(j)}} \bar{e}_{\eta}^{j}$ y appartient par le calcul ci-dessus, et que $\left(e-c^{(j)}\right)+p c^{(j-1)}=e\left(c_{f-j}+1\right)>e$, de sorte que l'on a encore $h_{j}=e-c^{(j)}$. Par (19) et le calcul du premier cas, on obtient comme annoncé :

$$
\varphi_{1}\left(u^{e-c^{(j)}} e^{j}\right)=\varphi_{1}\left(u^{e-c^{(j)}} \bar{e}_{\eta}^{j}\right)=-\bar{a}_{j} e^{j+1} .
$$


(d) Supposons que $j \in I_{\eta^{\prime}}^{\times}\left(\right.$et $\left.\eta_{j}=\eta\right)$. On déduit alors de (12) que :

$\operatorname{Fil}^{1} \overline{\mathcal{M}}^{j}=\bar{S} u^{e} \bar{e}_{\eta}^{j} \oplus \bar{S}\left(\bar{e}_{\eta^{\prime}}^{j}+\bar{a}_{j} u^{e-c^{(j)}} \bar{e}_{\eta}^{j}\right), \quad \varphi_{1}\left(u^{e} \bar{e}_{\eta}^{j}\right)=\bar{e}_{\eta}^{j+1}, \quad \varphi_{1}\left(\bar{e}_{\eta^{\prime}}^{j}+\bar{a}_{j} u^{e-c^{(j)}} \bar{e}_{\eta}^{j}\right)=\bar{e}_{\eta^{\prime}}^{j+1}$.

Dans le premier cas de (18), on vérifie que $h_{j}=e$ et $\varphi_{1}\left(u^{e} e^{j}\right)=\varphi_{1}\left(u^{e} \bar{e}_{\eta}^{j}\right)=\bar{e}_{\eta}^{j+1}=$ $e^{j+1}$. Dans le deuxième cas de (18), on vérifie de même que $h_{j}=e$ et $\varphi_{1}\left(u^{e} e^{j}\right)=$ $\varphi_{1}\left(u^{e} \bar{e}_{\eta}^{j}\right)=e^{j+1} \operatorname{par}(19)$.

Les énoncés de (17) sont donc vérifiés. Si l'on résume, on obtient d'après [15, Ex. 3.7] que :

$$
\operatorname{Hom}_{\mathrm{Fil}^{1}, \varphi_{1}}\left(\overline{\mathcal{N}}, \widehat{A}_{\text {cris }} \otimes_{\mathbb{Z}_{p}} \mathbb{F}_{p}\right)^{\vee}(1) \cong \operatorname{nr}(A) \overline{\eta_{0}} \omega_{\sigma_{0}}^{h},
$$

où $h:=\frac{1}{e} \sum_{j=0}^{f-1} p^{f-j} h_{j}$ et

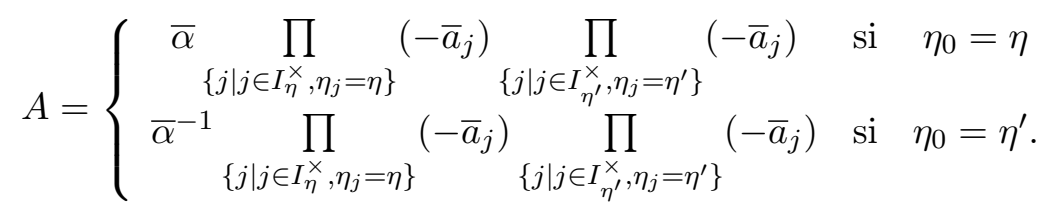

La formule annoncée pour $A$ s'en déduit en notant que (par la récurrence (14))

$$
\left\{j \mid j \in I_{\eta}^{\times}, \eta_{j}=\eta\right\} \coprod\left\{j \mid j \in I_{\eta^{\prime}}^{\times}, \eta_{j}=\eta^{\prime}\right\}=\left\{j \mid \eta_{j} \neq \eta_{j+1}\right\}
$$

et que $\left|\left\{j \mid \eta_{j} \neq \eta_{j+1}\right\}\right|$ est toujours pair. En regardant enfin le déterminant de $\bar{\rho}_{\mathcal{M}}$, on trouve que

$$
\begin{aligned}
\bar{\rho}_{\mathcal{M}} & \cong\left(\begin{array}{cc}
\operatorname{nr}(A) \overline{\eta_{0}} \omega_{\sigma_{0}}^{h} & * \\
0 & \operatorname{nr}\left(A^{-1}\right) \overline{\eta_{0}^{\mathrm{c}}} \omega_{\sigma_{0}}^{-h} \omega
\end{array}\right) \\
& \cong\left(\begin{array}{cc}
\operatorname{nr}(A) \overline{\eta_{0}}\left(\overline{\eta_{0}^{\mathrm{c}}}\right)^{-1} \omega_{\sigma_{0}}^{2 h} \omega^{-1} & * \\
0 & \operatorname{nr}\left(A^{-1}\right)
\end{array}\right) \otimes\left(\overline{\eta_{0}^{\mathrm{c}}} \omega_{\sigma_{0}}^{-h} \omega\right)
\end{aligned}
$$

où l'on a posé $\eta_{0}^{\mathrm{c}}:=\eta^{\prime}$ (resp. $\left.\eta_{0}^{\mathrm{c}}:=\eta\right)$ si $\eta_{0}=\eta\left(\right.$ resp. $\left.\eta_{0}=\eta^{\prime}\right)$. Un calcul analogue à celui mené dans la preuve de [5, Lem.6.3] donne les valeurs de $r_{j}^{\prime}$ listées dans (15) et achève donc la preuve. (Notons que seul le cas $\eta_{0}=\eta$ est traité dans [5, Lem.6.3], mais on trouve le même résultat lorsque $\eta_{0}=\eta^{\prime}$.)

Enfin, l'appartenance de $r_{j}^{\prime}$ à $\{0, \cdots, p-3\}$ se déduit de (15) et de (17), en utilisant [5, Lem.6.3].

Remarque 3.3. Lorsque $\bar{\rho}_{\mathcal{M}}$ est réductible scindée, on a a priori deux choix possibles pour la suite $\left\{\eta_{j}\right\}_{j}$. Chacun d'eux donnera une expression de $\bar{\rho}_{\mathcal{M}}$ analogue à celle de la proposition 3.2. Cela explique pourquoi nous aurons souvent besoin de traiter séparément le cas scindé (voir le corollaire 4.2 ci-après).

La preuve de la proposition suivante est essentiellement celle de [7, Prop. 5.1.2].

Proposition 3.4. Soit $\mathcal{M}$ un $\mathcal{O}_{E}$-module fortement divisible de type $\eta \otimes \eta^{\prime}$ et soit $\left\{\eta_{j}\right\}_{j}$ une suite vérifiant la récurrence (14). Soient $\left\{r_{j}^{\prime}\right\}_{0 \leq j \leq f-1}, \theta$ et $A$ comme dans l'énoncé de la proposition 3.2. Le module de Fontaine-Laffaille de $\bar{\rho}_{\mathcal{M}} \otimes \theta^{-1}$ est alors égal à :

$$
M=M^{\sigma_{0}} \times M^{\sigma_{0} \circ \varphi^{-1}} \times \cdots \times M^{\sigma_{0} \circ \varphi^{-(f-1)}},
$$


où l'on pose, en écrivant $M^{j}=M^{\sigma_{0} \circ \varphi^{-j}}$ et $a_{j}=a_{\sigma_{0} \circ \varphi^{-j}}$,

$$
\begin{aligned}
& \left\{\begin{array}{lccl}
M^{j} & = & k_{E} v^{j} \oplus k_{E} w^{j} & \\
\mathrm{Fil}^{i} M^{j} & = & M^{j} & \text { si } i \leq 0 \\
\mathrm{Fil}^{i} M^{j} & = & k_{E} w^{j} & \text { si } 1 \leq i \leq r_{f-j}^{\prime}+1 \\
\mathrm{Fil}^{i} M^{j} & = & 0 & \text { si } i \geq r_{f-j}^{\prime}+2
\end{array}\right. \\
& \left\{\begin{array}{ll}
\varphi\left(v^{j}\right) & =v^{j} \\
\varphi_{r_{f-j}^{\prime}+1}\left(w^{j}\right) & =w^{j+1}-A_{j} v^{j+1}
\end{array} \quad \text { si } 0 \leq j \leq f-2\right. \\
& \begin{cases}\varphi\left(v^{f-1}\right) & =A v^{0} \\
\varphi_{r_{1}^{\prime}+1}\left(w^{f-1}\right) & =A^{-1} w^{0}-A_{f-1} v^{0}\end{cases} \\
& \left\{\begin{array}{c}
A_{j}=\bar{a}_{j} \prod_{\substack{0 \leq i \leq j-1 \\
\left\{i \mid \eta_{i} \neq \eta_{i+1}\right\}}}\left(-\bar{a}_{i}^{-2}\right) \quad \text { si } \quad \eta_{j}=\eta_{j+1} \\
A_{j}=\bar{a}_{j}^{-1} \prod_{\substack{0 \leq i \leq j-1 \\
\left\{i \mid \eta_{i} \neq \eta_{i+1}\right\}}}\left(-\bar{a}_{i}^{-2}\right) \quad \text { si } \quad \eta_{j} \neq \eta_{j+1}
\end{array}\right.
\end{aligned}
$$

et où l'on doit multiplier le membre de droite par A lorsque $j=f-1$.

Démonstration. C'est la même preuve que celle de [7, Prop. 5.1.2] (ou de [6, Prop.2.3.1]), en notant que l'ensemble $I^{\times}$défini dans ladite preuve (ou $F(J)$ dans [6]) n'est autre que $\left\{j \mid \eta_{j} \neq \eta_{j+1}\right\}$.

\section{Valeurs spéciales de paramètres}

Nous démontrons maintenant le résultat principal de l'article, à savoir le théorème 4.7, qui donne une valeur spéciale du paramètre $x(\lambda)$ (défini dans la proposition 2.6) attachée à la représentation $\bar{\rho}$ de départ.

On fixe $\bar{\rho}: \operatorname{Gal}\left(\overline{\mathbb{Q}}_{p} / L\right) \rightarrow \mathrm{GL}_{2}\left(k_{E}\right)$ une représentation de la forme $(1)$ et $\lambda \in \mathcal{P D}\left(x_{0}, \cdots, x_{f-1}\right)$ un $f$-uplet vérifiant la condition (6). Soit $\chi: I\left(\mathcal{O}_{L}\right) \rightarrow k_{E}^{\times}$le caractère qui lui correspond, i.e. tel que $\chi \cong \tau(\lambda)^{I_{1}\left(\mathcal{O}_{L}\right)}$, que l'on écrit (de manière unique) sous la forme $\chi=\bar{\eta}(\lambda) \otimes \bar{\eta}^{\prime}(\lambda)$ avec $\bar{\eta}(\lambda), \bar{\eta}^{\prime}(\lambda): \mathcal{O}_{L}^{\times} \rightarrow k_{E}^{\times}$des caractères lisses. On pose

$$
\eta(\lambda):=[\bar{\eta}(\lambda)] \quad\left(\text { resp. } \eta^{\prime}(\lambda):=\left[\bar{\eta}^{\prime}(\lambda)\right]\right)
$$

les relèvements de $\eta(\lambda)$ et $\eta^{\prime}(\lambda)$ à $\mathcal{O}_{E}^{\times}$via le représentant multiplicatif $[\cdot]: k_{E}^{\times} \rightarrow \mathcal{O}_{E}^{\times}$. On vérifie que $\eta(\lambda)=\eta^{\prime}(\lambda) \prod_{j=0}^{f-1}\left[\sigma_{0}\right]^{p^{j} c_{j}}$ avec $c_{j}:=\lambda_{j}\left(r_{j}\right)$.

Si $J$ est un sous-ensemble de $\mathcal{S}$, on définit la "frontière" $F(J)$ de $J$ comme dans $[6,(5)]$ :

$$
F(J):=\{j \in \mathcal{S} \mid j \in J, j+1 \notin J\} \coprod\{j \in \mathcal{S} \mid j \notin J, j+1 \in J\} .
$$

Corollaire 4.1. Conservons les notations précédentes et supposons que $\bar{\rho}$ est non scindée. Soit $\mathcal{M}$ un $\mathcal{O}_{E}$-module fortement divisible de type $\eta(\lambda) \otimes \eta^{\prime}(\lambda)$ tel que $\bar{\rho} \cong \bar{\rho}_{\mathcal{M}}$. Définissons $\left\{\eta_{j}\right\}_{j}$ comme l'unique suite à valeur dans $\eta_{j} \in\left\{\eta(\lambda), \eta^{\prime}(\lambda)\right\}$ vérifiant la récurrence (14). Alors

(i) $\eta_{j}=\eta(\lambda)\left(\right.$ resp. $\left.\eta_{j}=\eta^{\prime}(\lambda)\right)$ si et seulement si $f+1-j \notin I(\lambda)($ resp. $f+1-j \in I(\lambda))$, où $I(\lambda)$ est défini par (9);

(ii) $\eta_{j} \neq \eta_{j+1}$ si et seulement si $f-j \in F(I(\lambda))$;

(iii) $j \in I_{\eta(\lambda)}$ si et seulement si $f-j \in \delta^{-1}\left(J^{\mathrm{min}}\right)$; en particulier, on a $\left|I_{\eta(\lambda)}\right|=\left|J^{\mathrm{min}}\right|$ et $I_{\eta^{\prime}(\lambda)}=\left|\mathcal{S} \backslash J^{\mathrm{min}}\right|$. 
Démonstration. Puisque $\bar{\rho}$ est supposée non scindée, la condition $\bar{\rho} \cong \bar{\rho}_{\mathcal{M}}$ implique que $r_{f-j}=r_{f-j}^{\prime}$ pour tout $j$.

(i) Sachant que $c_{j}=\lambda_{j}\left(r_{j}\right)$, le corollaire 2.3 et la proposition 3.2 assurent que :

$$
\begin{aligned}
\eta_{j}=\eta(\lambda) & \Longleftrightarrow \lambda_{f-j}\left(x_{f-j}\right) \in\left\{x_{f-j}, x_{f-j}+1, x_{f-j}+2\right\} \\
& \Longleftrightarrow f-j+1 \notin I(\lambda),
\end{aligned}
$$

ce qui prouve (i). Dans le cas où $r_{f-j} \in\left\{\frac{p-1}{2}, \frac{p-3}{2}, \frac{p-5}{2}\right\}$, une analyse plus poussée sur le déterminant est nécessaire, voir la preuve de [8, Lem.12.8].

(ii) Il se déduit du (i).

(iii) $\operatorname{Par}(15), j \in I_{\eta(\lambda)}$ si et seulement si $c_{f-j} \in\left\{r_{f-j}+1, r_{f-j}+2, p-1-r_{f-j}\right\}$, si et seulement si $f-j \in \delta^{-1}\left(J^{\text {min }}\right)$ d'après le corollaire 2.2.

Corollaire 4.2. Conservons les notations précédentes et supposons que $\bar{\rho}$ est scindée. Soit $\mathcal{M}$ un $\mathcal{O}_{E}$-module fortement divisible de type $\eta(\lambda) \otimes \eta^{\prime}(\lambda)$ tel que $\bar{\rho} \cong \bar{\rho}_{\mathcal{M}}$. Alors $\left|I_{\eta(\lambda)}\right|=\left|J^{\mathrm{min}}\right|$, $\left|I_{\eta^{\prime}(\lambda)}\right|=\left|\mathcal{S} \backslash J^{\min }\right|$ et

(i) $\bar{\alpha}=\xi$ si $\lambda_{j}\left(x_{j}\right) \in\left\{x_{j}, x_{j}+2\right\}$ pour tout $j$;

(ii) $\bar{\alpha}=\xi^{-1}$ si $\lambda_{j}\left(x_{j}\right) \in\left\{p-1-x_{j}, p-3-x_{j}\right\}$ pour tout $j$.

Démonstration. Reprenons les notations de la proposition 3.2. Puisque $\bar{\rho}_{\mathcal{M}}$ est réductible scindée, il y a deux possibilités pour la suite $\left\{\eta_{j}\right\}_{j}$ : soit la suite constante $\{\eta(\lambda)\}_{j}$, soit la suite constante $\left\{\eta^{\prime}(\lambda)\right\}_{j}$. La proposition 3.2 nous donne donc deux façons d'écrire $\bar{\rho}_{\mathcal{M}}$.

(a) Si $\eta_{j}=\eta(\lambda)$ pour tout $j$, alors $\bar{\rho}_{\mathcal{M}}$ s'écrit sous la forme

$$
\bar{\rho}_{\mathcal{M}} \cong\left(\operatorname{nr}(\bar{\alpha}) \omega_{\sigma_{0}}^{\sum_{j=0}^{f-1} p^{j}\left(r_{j}^{\prime}+1\right)} \oplus \operatorname{nr}\left(\bar{\alpha}^{-1}\right)\right) \otimes \theta^{\prime}
$$

avec $\theta^{\prime}$ caractère convenable uniquement déterminé et, sachant que $I_{\eta(\lambda)}^{\times}$et $I_{\eta^{\prime}(\lambda)}^{\times}$sont tous deux vides,

$$
\left\{\begin{array}{lll}
r_{f-j}^{\prime}=c_{f-j}-2 & \text { si } & j \in I_{\eta(\lambda)}^{0} \\
r_{f-j}^{\prime}=c_{f-j} & \text { si } & j \in I_{\eta^{\prime}(\lambda)}^{0}
\end{array} .\right.
$$

(b) Si $\eta_{j}=\eta^{\prime}(\lambda)$ pour tout $j$, alors $\bar{\rho}_{\mathcal{M}}$ s'écrit sous la forme

$$
\bar{\rho}_{\mathcal{M}} \cong\left(\operatorname{nr}\left(\bar{\alpha}^{-1}\right) \omega_{\sigma_{0}}^{\sum_{j=0}^{f-1} p^{j}\left(r_{j}^{\prime \prime}+1\right)} \oplus \operatorname{nr}(\bar{\alpha})\right) \otimes \theta^{\prime \prime}
$$

avec $\theta^{\prime \prime}$ caractère convenable uniquement déterminé et

$$
\left\{\begin{array}{lll}
r_{f-j}^{\prime \prime}=p-1-c_{f-j} & \text { si } & j \in I_{\eta(\lambda)}^{0} \\
r_{f-j}^{\prime \prime}=p-3-c_{f-j} & \text { si } & j \in I_{\eta^{\prime}(\lambda)}^{0}
\end{array} .\right.
$$

Montrons maintenant le corollaire. (i) Supposons que $\lambda_{j}\left(x_{j}\right) \in\left\{x_{j}, x_{j}+2\right\}$, auquel cas on a $c_{j}=\lambda_{j}\left(r_{j}\right) \in\left\{r_{j}, r_{j}+2\right\}$. Dans ce cas, la condition $\bar{\rho} \cong \bar{\rho}_{\mathcal{M}}$ force que l'on est dans le cas (a) ci-dessus, c'est-à-dire :

$$
\theta^{\prime}=1, \quad \bar{\alpha}=\xi, \quad r_{j}^{\prime}=r_{j} .
$$

(ii) Supposons que $\lambda_{j}\left(x_{j}\right) \in\left\{p-1-x_{i}, p-3-x_{i}\right\}$, auquel cas $c_{j}=\lambda_{j}\left(r_{j}\right) \in\left\{p-1-r_{j}, p-\right.$ $\left.3-r_{j}\right\}$. Dans ce cas, la condition $\bar{\rho} \cong \bar{\rho}_{\mathcal{M}}$ force que l'on est dans le cas (b) ci-dessus, donc

$$
\theta^{\prime \prime}=1, \quad \bar{\alpha}=\xi^{-1}, \quad r_{j}^{\prime \prime}=r_{j} .
$$


Enfin, dans les deux cas, on a $j \in I_{\eta(\lambda)}$ si et seulement si $r_{f-j} \in\left\{c_{f-j}-2, p-1-c_{f-j}\right\}$, si et seulement si $f-j \in \delta^{-1}\left(J^{\text {min }}\right)$ par définition de $J^{\text {min }}$. On a donc $\left|I_{\eta(\lambda)}\right|=\left|J^{\text {min }}\right|$, et par conséquent $\left|I_{\eta^{\prime}(J)}\right|=\left|\mathcal{S} \backslash J^{\mathrm{min}}\right|$.

Théorème 4.3. Conservons les notations du début de cette section et considérons $\mathcal{M}$ un $\mathcal{O}_{E}$-module fortement divisible de type $\eta(\lambda) \otimes \eta^{\prime}(\lambda)$ vérifiant $\bar{\rho} \cong \bar{\rho}_{\mathcal{M}}$. Soit $G$ le groupe $p$ divisible avec donnée de descente correspondant à $\mathcal{M}$ et $D$ le module de Dieudonné contravariant associé à $G$. Alors la valeur propre de $\varphi^{f}$ sur la partie isotypique de $D$ pour le caractère $\eta^{\prime}(\lambda)$ de $\operatorname{Gal}(L[\sqrt[e]{-p}] / L)$ est égale à $p^{\left|\mathcal{S} \backslash J^{\mathrm{min}}\right|} \alpha^{\prime}$ où $\alpha^{\prime} \in \mathcal{O}_{E}^{\times}$a la réduction suivante dans $k_{E}^{\times}:$

$$
\bar{\alpha}^{\prime}=(-1)^{\frac{1}{2}|F(I(\lambda))|} \frac{\sqrt[f]{\xi}^{|I(\lambda)|}}{\sqrt[f]{\xi}^{|\mathcal{S} \backslash I(\lambda)|}} \frac{\prod_{\{j \mid j \in I(\lambda), j+1 \notin I(\lambda)\}} \mu_{f-j}}{\prod_{\{j \mid j \notin I(\lambda), j+1 \in I(\lambda)\}} \mu_{f-j}} .
$$

Démonstration. Remarquons que l'élément de $k_{E}^{\times}$défini en (20) ne dépend pas du choix de $\sqrt[f]{\xi}$. En effet, le changement de $\sqrt[f]{\xi}$ par $\mu \sqrt[f]{\xi}$ avec $\mu \in k_{E}^{\times}$et $\mu^{f}=1$ modifie les éléments $\left\{\mu_{i}\right\}_{0 \leq i \leq f-1}$ via la transformation suivante :

$$
\mu_{i} \mapsto \mu^{2(i+1)} \mu_{i}
$$

de sorte que la formule (20) est multipliée par

$$
\frac{\mu^{|I(\lambda)|}}{\mu^{|\mathcal{S} \backslash I(\lambda)|}} \frac{\prod_{\{j \mid j \in I(\lambda), j+1 \notin I(\lambda)\}} \mu^{2(f-j+1)}}{\prod_{\{j \mid j \notin I(\lambda), j+1 \in I(\lambda)\}} \mu^{2(f-j+1)}}=\mu^{2|I(\lambda)|} \cdot \frac{\prod_{\{j \mid j \notin I(\lambda), j+1 \in I(\lambda)\}} \mu^{2 j}}{\prod_{\{j \mid j \in I(\lambda), j+1 \notin I(\lambda)\}} \mu^{2 j}}
$$

qui vaut 1 étant donné que $|\{j \mid j \in I(\lambda), j+1 \notin I(\lambda)\}|=|\{j \mid j \notin I(\lambda), j+1 \in I(\lambda)\}|$ et que $\sum_{\{j \mid j \notin I(\lambda), j+1 \in I(\lambda)\}} j-\sum_{\{j \mid j \in I(\lambda), j+1 \notin I(\lambda)\}} j=|\mathcal{S} \backslash I(\lambda)|$.

La définition de $\mathcal{M}$ implique ensuite que la valeur propre de $\varphi^{f}$ sur la partie isotypique de $D$ pour le caractère $\eta^{\prime}(\lambda)$ est égal à $p^{\left|I_{\eta^{\prime}(\lambda)}\right|} \alpha^{\prime}$, où $\alpha^{\prime}$ est comme décrit dans l'expression de $\mathcal{M}$ au début du $\S 3$. Puisque les corollaires 4.1 et 4.2 assurent que $\left|I_{\eta^{\prime}(\lambda)}\right|=\left|\mathcal{S} \backslash J^{\mathrm{min}}\right|$, il reste à calculer la réduction dans $k_{E}^{\times}$de $\alpha^{\prime}$.

Supposons d'abord que $\bar{\rho} \cong \bar{\rho}_{\mathcal{M}}$ soit scindée. Il y a donc deux possibilités pour $\lambda$ :

(i) si $\lambda_{j} \in\left\{x_{j}, x_{j}+2\right\}$ pour tout $j$, on a alors $\bar{\alpha}^{\prime}=\xi^{-1}$ par le corollaire 4.2 , puis $I(\lambda)=\emptyset$ et $F(I(\lambda))=\emptyset$ par (9), d'où la formule annoncée;

(ii) si $\lambda_{i}\left(x_{j}\right) \in\left\{p-1-x_{j}, p-3-x_{j}\right\}$ pour tout $j$, on a alors $\bar{\alpha}^{\prime}=\xi$ par le corollaire 4.2 , puis $I(\lambda)=\mathcal{S}$ et $F(I(\lambda))=\emptyset$ par (9), d'où la formule annoncée.

Supposons maintenant que $\bar{\rho}$ soit non scindée et notons $\left\{\eta_{j}\right\}_{j}$ l'unique suite (avec $\eta_{j} \in$ $\left.\left\{\eta(\lambda), \eta^{\prime}(\lambda)\right\}\right)$ associée à $\mathcal{M}$. Comme $\bar{\rho}$ est non scindée, la condition $\bar{\rho} \cong \bar{\rho}_{\mathcal{M}}$ nécessite que l'on ait (avec les notations de la proposition 3.2) :

$$
\theta=1, \quad A=\xi, \quad r_{j}^{\prime}=r_{j}, \forall 0 \leq j \leq f-1 .
$$

On obtient en particulier

$$
\bar{\alpha}^{\prime}=\bar{\alpha}^{-1}=\left\{\begin{array}{lll}
\xi^{-1} \prod_{\left\{j \mid \eta_{j} \neq \eta_{j+1}\right\}} \bar{a}_{j} & \text { si } & \eta_{0}=\eta(\lambda) \\
\xi\left(\prod_{\left\{j \mid \eta_{j} \neq \eta_{j+1}\right\}} \bar{a}_{j}\right)^{-1} & \text { si } & \eta_{0}=\eta^{\prime}(\lambda)
\end{array}\right.
$$

ce qui ramène à calculer $\prod_{\left\{j \mid \eta_{j} \neq \eta_{j+1}\right\}} \bar{a}_{j}$ pour terminer la preuve. 
En comparant le module de Fontaine-Laffaille de $\bar{\rho}_{\mathcal{M}}$ donné par la proposition 3.4 à celui de $\bar{\rho}$ normalisé au $\S 2$, on obtient comme dans la preuve de [6, Thm.2.4.1] que

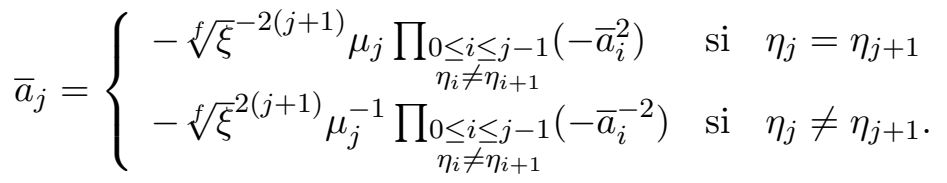

En utilisant $(21)$ et en notant $0 \leq j_{1}<j_{2}<\cdots<j_{2 t-1}<j_{2 t} \leq f-1$ les éléments de $\{0, \cdots, f-1\}$ pour lesquels $\eta_{j} \neq \eta_{j+1}$ (remarquons que $\left|\left\{j \mid \eta_{j} \neq \eta_{j+1}\right\}\right|$ est toujours pair), on montre comme dans la preuve de [6, Thm.2.4.1] que :

$$
\bar{a}_{j_{1}} \bar{a}_{j_{2}} \cdots \bar{a}_{j_{2 t}}=\prod_{\left\{j \mid \eta_{j} \neq \eta_{j+1}\right\}} \bar{a}_{j}=(-1)^{t} \frac{\prod_{i=1}^{t} \mu_{j_{2 i-1}} \sqrt[f]{\xi}^{2 j_{2 i}}}{\prod_{i=1}^{t} \mu_{j_{2 i}} \sqrt[f]{\xi}^{2 j_{2 i-1}}} .
$$

(Dans la preuve loc. cit., $\frac{\alpha_{i}}{\beta_{i}}$ correspond à notre $(\sqrt[f]{\xi})^{2}, F(J)$ à notre $\left\{j \mid \eta_{j} \neq \eta_{j+1}\right\}$, et $x_{j}$ à notre $\mu_{j}$.)

Supposons tout d'abord que $\eta_{0}=\eta(\lambda)$. On a alors les égalités suivantes :

$$
\begin{gathered}
\left\{j_{2 i-1}, 1 \leq i \leq t\right\}=\left\{j \mid \eta_{j}=\eta(\lambda), \eta_{j+1}=\eta^{\prime}(\lambda)\right\} \\
\left\{j_{2 i}, 1 \leq i \leq t\right\}=\left\{j \mid \eta_{j}=\eta^{\prime}(\lambda), \eta_{j+1}=\eta(\lambda)\right\}
\end{gathered}
$$

et

$$
\sum_{i=1}^{t}\left(j_{2 i}-j_{2 i-1}\right)=\left|\left\{j \mid \eta_{j}=\eta^{\prime}(\lambda)\right\}\right| .
$$

On déduit donc de tout ce qui précède que $\left(\right.$ si $\left.\eta_{0}=\eta(\lambda)\right)$ :

$$
\begin{aligned}
& \bar{\alpha}^{\prime}=\xi^{-1}\left(\prod_{\left\{j \mid \eta_{j} \neq \eta_{j+1}\right\}} \bar{a}_{j}\right) \\
& =\frac{1}{\sqrt[f]{\xi^{f}}} \cdot(-1)^{\frac{1}{2}\left|\left\{j \mid \eta_{j} \neq \eta_{j+1}\right\}\right|} \sqrt[f]{\xi^{2\left|\left\{j \mid \eta_{j}=\eta^{\prime}(\lambda)\right\}\right|} \frac{\prod_{\left\{j \mid \eta_{j}=\eta(\lambda), \eta_{j+1}=\eta^{\prime}(\lambda)\right\}} \mu_{j}}{\prod_{\left\{j \mid \eta_{j}=\eta^{\prime}(\lambda), \eta_{j+1}=\eta(\lambda)\right\}} \mu_{j}}}
\end{aligned}
$$

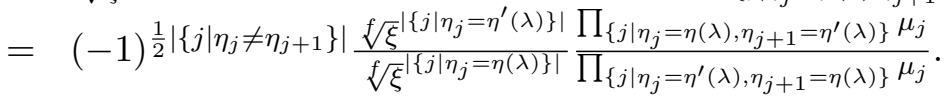

La formule voulue s'en déduit à l'aide du corollaire 4.1 qui nous dit que

$$
\left|\left\{j \mid \eta_{j} \neq \eta_{j+1}\right\}\right|=|F(I(\lambda))|, \quad\left|\left\{j \mid \eta_{j}=\eta(\lambda)\right\}\right|=|\mathcal{S} \backslash I(\lambda)|, \quad\left|\left\{j \mid \eta_{j}=\eta^{\prime}(\lambda)\right\}\right|=|I(\lambda)|
$$

et que $\left\{j \mid \eta_{j}=\eta(\lambda), \eta_{j+1}=\eta^{\prime}(\lambda)\right\}=\{f-j \mid j \in I(\lambda), j+1 \notin I(\lambda)\}$ et $\left\{j \mid \eta_{j}=\eta^{\prime}(\lambda), \eta_{j+1}=\right.$ $\eta(\lambda)\}=\{f-j \mid j \notin I(\lambda), j+1 \in I(\lambda)\}$. On laisse le lecteur traiter le cas $\eta_{0}=\eta^{\prime}(\lambda)$ qui mène la même formule.

Soit $\tau \in \mathcal{D}(\bar{\rho})$ le poids de Serre pour lequel $\chi$ et $\chi^{s}$ apparaissent tous les deux sur $D_{\tau}(\bar{\rho})^{I_{1}\left(\mathcal{O}_{L}\right)}$, avec $\chi=\tau(\lambda)^{I_{1}\left(\mathcal{O}_{L}\right)}$. En notant $s_{j}=\tau_{j}\left(r_{j}\right)$, on a donc

$$
\tau=\left(s_{0}, \cdots, s_{f-1}\right) \otimes \operatorname{det}^{e(\tau)\left(r_{0}, \cdots, r_{f-1}\right)} .
$$

Le prochain lemme permet d'écrire $c_{j}=\lambda_{j}\left(r_{j}\right)(j \in \mathcal{S})$ en fonction de $s_{j}$. 
Lemme 4.4. (i) On a les relations suivantes:

$$
\begin{array}{ccclll}
c_{j}=p-2-s_{j} & \text { si } & j \in J^{\min } & \text { et } & j-1 \notin J^{\min } \\
c_{j}=p-1-s_{j} & \text { si } & j \in J^{\min } & \text { et } & j-1 \in J^{\mathrm{min}} \\
c_{j}= & s_{j}+1 & \text { si } & j \notin J^{\mathrm{min}} & \text { et } & j-1 \in J^{\mathrm{min}} \\
c_{j}= & s_{j} & \text { si } & j \notin J^{\mathrm{min}} & \text { et } & j-1 \notin J^{\mathrm{min}}
\end{array}
$$

(ii) On a

$$
\prod_{j \notin J^{\min }}\left[\sigma_{0}\right]^{p^{j}\left(p-1-s_{j}\right)} \prod_{j \in \mathcal{S}}\left[\sigma_{0}\right]^{p^{j} c_{j}}=\prod_{j \in J^{\min }}\left[\sigma_{0}\right]^{p^{j}\left(p-1-s_{j}\right)} .
$$

Démonstration. Le (i) se déduit du fait que $\tau$ apparaît dans $\bar{\sigma}\left(\chi^{s}\right)=\operatorname{Ind}_{I\left(\mathcal{O}_{L}\right)}^{\mathrm{GL}_{2}\left(\mathcal{O}_{L}\right)} \chi^{s}$ en "position" $J^{\min }$ (voir [8, Lem.2.7]) et le (ii) se déduit du (i).

Théorème 4.5. Soient $\mathcal{M}, G$ et $D$ comme dans le théorème 4.3. Soit $V$ (resp. $V^{\prime}$ ) la valeur propre de $\varphi^{f}$ sur la partie $\eta(\lambda)$-isotypique (resp. $\eta^{\prime}(\lambda)$-isotypique) de D. Considérons la série principale modérément ramifiée :

$$
\Pi(\lambda):=\operatorname{Ind}_{B(L)}^{\mathrm{GL}_{2}(L)} \eta^{\prime}(\lambda) \operatorname{nr}\left(V^{\prime}\right)|\cdot| \otimes \eta(\lambda) \operatorname{nr}(V)
$$

où $\eta(\lambda)$ et $\eta^{\prime}(\lambda)$ sont vus comme des caractères de $L^{\times}$envoyant $p$ et $1+p \mathcal{O}_{L}$ sur 1 . Soit $\hat{v}(\lambda) \in \Pi(\lambda)^{I_{1}\left(\mathcal{O}_{L}\right)}$ un vecteur non nul sur lequel $I\left(\mathcal{O}_{L}\right)$ agit par $\eta^{\prime}(\lambda) \otimes \eta(\lambda)$. Si $J^{\min } \notin\{\emptyset, \mathcal{S}\}$, il existe un unique élément $\hat{x}(\lambda) \in \mathcal{O}_{E}^{\times}$tel que l'on ait l'égalité suivante dans $\Pi(\lambda)$ :

$$
\begin{aligned}
\sum_{t \in \mathbb{F}_{q}}\left(\prod_{j \notin J^{\min }}\left[\sigma_{0}(t)\right]^{p^{j}\left(p-1-s_{j}\right)}\right)\left(\begin{array}{cc}
{[t]} & 1 \\
1 & 0
\end{array}\right) & \left(\begin{array}{ll}
0 & 1 \\
p & 0
\end{array}\right) \hat{v}(\lambda) \\
& =\hat{x}(\lambda) \sum_{t \in \mathbb{F}_{q}}\left(\prod_{j \in J^{\min }}\left[\sigma_{0}(t)\right]^{p^{j}\left(p-1-s_{j}\right)}\right)\left(\begin{array}{cc}
{[t]} & 1 \\
1 & 0
\end{array}\right) \hat{v}(\lambda) .
\end{aligned}
$$

De plus, la réduction de $\hat{x}(\lambda)$ dans $k_{E}^{\times}$est égale $\grave{a}$

$$
(-1)^{1+\frac{\left|F\left(J^{\mathrm{min}}\right)\right|}{2}+\frac{|F(I(\lambda))|}{2}} \frac{\sqrt[f]{\xi^{|I(\lambda)|}}}{\sqrt[f]{\xi}|\mathcal{S} \backslash I(\lambda)|} \frac{\prod_{\left\{j \mid j \notin J^{\min }, j-1 \in J^{\min }\right\}}\left(s_{j}+1\right)}{\prod_{\left\{j \mid j \in J^{\min }, j-1 \notin J^{\min }\right\}}\left(s_{j}+1\right)} \frac{\prod_{\{j \mid j \in I(\lambda), j+1 \notin I(\lambda)\}} \mu_{f-j}}{\prod_{\{j \mid j \notin I(\lambda), j+1 \in I(\lambda)\}} \mu_{f-j}} .
$$

Démonstration. Notons tout d'abord que le vecteur $\hat{v}(\lambda)$ existe et est unique à un scalaire près. La preuve de $\left[6\right.$, Thm. 2.5.2] et le lemme 4.4 montrent (puisque $J^{\min } \notin\{\emptyset, \mathcal{S}\}$ ) que l'on a

$$
\hat{x}(\lambda)=(-1)^{\sum_{j \notin J} \min s_{j}} \frac{1}{p^{f}} V^{\prime} X^{\prime}
$$

$\operatorname{avec} X^{\prime}=U p^{\left|J^{\min }\right|}+C \in \mathcal{O}_{E}^{\times}, \operatorname{val}(C)>\left|J^{\min }\right|$ et

$$
U \equiv(-1)^{1+\frac{\left|F\left(J^{\mathrm{min}}\right)\right|}{2}+\sum_{j \notin J^{\min }} s_{j}} \frac{\prod_{\left\{j \notin J^{\min }, j-1 \in J^{\min }\right\}}\left(s_{j}+1\right)}{\prod_{\left\{j \in J^{\min }, j-1 \notin J^{\min }\right\}}\left(s_{j}+1\right)}(\bmod p) .
$$

Nous rappelons que dans la preuve de [6, Thm. 2.5.2], $r_{\sigma_{0} \circ \varphi^{j}}$ correspond à notre $s_{j}$, et $J$ à $\mathcal{S} \backslash J^{\text {min }}$. Sachant que $V^{\prime}=p^{\left|\mathcal{S} \backslash J^{\mathrm{min}}\right|} \alpha^{\prime}$ d'après le théorème 4.3 , on en déduit que

$$
\begin{aligned}
\hat{x}(\lambda) & =(-1)^{j \notin J^{\min }}{ }^{s_{j}} \frac{1}{p^{f}} p^{\left|\mathcal{S} \backslash J^{\min }\right|} \alpha^{\prime}\left(U p^{\left|J^{\min }\right|}+C\right) \\
& \equiv(-1)^{j \notin J^{\min }} s^{\prime} \alpha^{\prime} U(\bmod p) \\
& \equiv \bar{\alpha}^{\prime}(-1)^{1+\frac{\left|F\left(J^{\min }\right)\right|}{2}} \frac{\prod_{\left\{j \mid j \notin J^{\min }, j-1 \in J^{\min }\right\}}\left(s_{j}+1\right)}{\prod_{\left\{j \mid j \in J^{\min }, j-1 \notin J^{\min }\right\}}\left(s_{j}+1\right)}(\bmod p),
\end{aligned}
$$


et l'on peut alors conclure en utilisant le théorème 4.3.

Remarque 4.6. Regardons les cas $J^{\mathrm{min}}=\emptyset$ ou $J^{\mathrm{min}}=\mathcal{S}$. D'après la corollaire 2.2 et la définition de $\mathcal{P} \mathcal{D}\left(x_{0}, \cdots, x_{f-1}\right)$, on doit alors avoir $J_{\tau} \in\{\emptyset, \mathcal{S}\}$. Le cas $J_{\tau}=\emptyset$ est couvert par [6, Rem.2.5.3]. Lorsque $J_{\tau}=\mathcal{S}, \bar{\rho}$ est forcément scindée, et l'on dispose alors du résultat suitant : si $J^{\mathrm{min}}=\emptyset$, alors

$$
\sum_{t \in \mathbb{F}_{q}}\left(\prod_{j \in \mathcal{S}}\left[\sigma_{0}(t)\right]^{p^{j}\left(p-1-s_{j}\right)}\right)\left(\begin{array}{cc}
{[t]} & 1 \\
1 & 0
\end{array}\right)\left(\begin{array}{ll}
0 & 1 \\
p & 0
\end{array}\right) \hat{v}(\lambda)=-\alpha^{\prime} \sum_{t \in \mathbb{F}_{q}}\left(\begin{array}{cc}
{[t]} & 1 \\
1 & 0
\end{array}\right) \hat{v}(\lambda)+q \alpha^{\prime}\left(\begin{array}{ll}
0 & 1 \\
1 & 0
\end{array}\right) \hat{v}(\lambda) ;
$$

si $J^{\min }=\mathcal{S}$, alors

$$
\sum_{t \in \mathbb{F}_{q}}\left(\begin{array}{cc}
{[t]} & 1 \\
1 & 0
\end{array}\right)\left(\begin{array}{ll}
0 & 1 \\
p & 0
\end{array}\right) \hat{v}(\lambda)=-\alpha \sum_{t \in \mathbb{F}_{q}}\left(\prod_{j \in \mathcal{S}}\left[\sigma_{0}(t)\right]^{p^{j}\left(p-1-s_{j}\right)}\right)\left(\begin{array}{cc}
{[t]} & 1 \\
1 & 0
\end{array}\right) \hat{v}(\lambda)+q\left(\begin{array}{cc}
p & 0 \\
0 & 1
\end{array}\right) \hat{v}(\lambda) .
$$

Tout ce qui précède nous permet maintenant d'obtenir notre résultat principal.

Théorème 4.7. Soient $\bar{\rho}$ et $\lambda \in \mathcal{P D}\left(x_{0}, \cdots, x_{f-1}\right)$ vérifiant la condition (6) et soit $\tau \in$ $\mathcal{D}(\bar{\rho})$ le poids de Serre associé. Ecrivons $s_{j}=\tau_{j}\left(r_{j}\right)$ pour tout $j$. Soient $\mathcal{M}$ un $\mathcal{O}_{E}$-module fortement divisible vérifiant les hypothèses du théorème 4.3 et $\Pi(\lambda)$ la représentation définie par (22). Soit $\pi$ une représentation lisse de $\mathrm{GL}_{2}(L)$ sur $k_{E}$ vérifiant les propriétés suivantes :

(i) $p \in L^{\times}$agit trivialement sur $\pi$

(ii) $\pi$ contient $D(\bar{\rho})$

(iii) $D(\bar{\rho})^{I_{1}\left(\mathcal{O}_{L}\right)}$ est stable sous l'action de $\left(\begin{array}{ll}0 & 1 \\ p & 0\end{array}\right)$ sur $\pi$.

Définissons $x(\lambda) \in k_{E}^{\times}$comme dans la proposition 2.6 et $\hat{v}(\lambda)$ comme dans le théorème 4.5. Supposons de plus qu'il existe un $\mathcal{O}_{E}$-réseau stable $\Pi^{0}(\lambda)$ dans $\Pi(\lambda)$ contenant $\hat{v}(\lambda)$ ainsi qu'un morphisme $\mathcal{O}_{E}$-linéaire $\mathrm{GL}_{2}(L)$-équivariant $\Pi^{0}(\lambda) \rightarrow \pi$ tel que l'image de $\hat{v}(\lambda)$ par ce morphisme soit un élément non nul de $D(\bar{\rho})$. Alors on a :

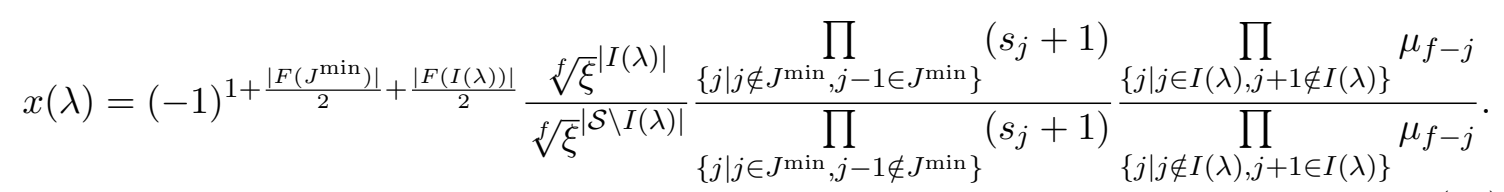

Démonstration. Cela découle de la proposition 2.6 et du théorème 4.5, car nos hypothèses assurent que la réduction modulo $p$ de $\hat{v}(\lambda)$ est forcément un multiple (non nul) de $v(\lambda)$.

Remarque 4.8. (i) L'hypothèse (iii) sur $\pi$ est en particulier satisfaite si l'on a l'égalité $D(\bar{\rho})^{I_{1}\left(\mathcal{O}_{L}\right)}=\pi^{I_{1}\left(\mathcal{O}_{L}\right)}$. Dans ce cas, l'image de $\hat{v}(\lambda)$ se trouve automatiquement dans $D(\bar{\rho})$.

(ii) Lorsque $J_{\tau}=\emptyset$, on a $J^{\mathrm{min}}=\mathcal{S} \backslash J$ et $I(\lambda)=\delta^{-1}(\mathcal{S} \backslash J)$, ce qui implique que $\left|F\left(J^{\mathrm{min}}\right)\right|=|F(I(\lambda))|$ et que $\frac{\left|F\left(J^{\mathrm{min}}\right)\right|+|F(I(\lambda))|}{2}$ est pair. On retrouve donc [6, Thm.2.5.2] à partir du théorème $4 . \%$.

On donnera au $\S 6$ des exemples lorsque $f=3$ pour expliciter les paramètres que nous avons calculé. 


\section{Paramètres cycliques}

On définit et calcule des paramètres cycliques de diagrammes de Diamond, toujours pour $\bar{\rho}$ réductible non scindée, qui sont analogues à ceux étudiés par Breuil dans [4].

Fixons $\bar{\rho}$ réductible comme en (1). Notons $\bar{\rho}^{\text {ss }}$ la semi-simplification de $\bar{\rho}$ et $\mathcal{D}\left(\bar{\rho}^{\text {ss }}\right)$ l'ensemble des poids de Serre qui lui sont associés. Soit $D\left(\bar{\rho}^{\mathrm{ss}}\right)$ la représentation de $\mathrm{GL}_{2}\left(\mathbb{F}_{q}\right)$ associée à $\bar{\rho}^{\mathrm{ss}}(\S 2)$. D'après $\left[8\right.$, Thm. 15.4], $D\left(\bar{\rho}^{\mathrm{ss}}\right)$ se décompose en somme directe $\oplus_{\ell=0}^{f} D_{\ell}\left(\bar{\rho}^{\mathrm{ss}}\right)$ vérifiant

$$
\operatorname{soc}_{\mathrm{GL}_{2}\left(\mathbb{F}_{q}\right)} D_{\ell}\left(\bar{\rho}^{\mathrm{ss}}\right)=\bigoplus_{J \subseteq \mathcal{S},|J|=\ell} \tau(J)
$$

où $\tau(J)$ désigne l'unique poids de $\mathcal{D}\left(\bar{\rho}^{\mathrm{ss}}\right)$ tel que $J_{\tau(J)}=J$ (cf. (4)). La proposition 5.2 ciaprès est une conséquence de la construction de $[8, \S 15]$ (mais pas énoncée explicitement). Commençons par un résultat préliminaire sur la structure de $D(\bar{\rho})$.

Lemme 5.1. Soit $J \subseteq \mathcal{S}$ et $\tau(J) \in \mathcal{D}\left(\bar{\rho}^{\mathrm{ss}}\right)$ le poids associé. Notons $W_{\tau(J)}$ l'unique sousreprésentation de $D_{\tau\left(J \cap J_{\bar{\rho}}\right)}(\bar{\rho}) \subset D(\bar{\rho})$ de co-socle $\tau(J)$. Alors $W_{\tau(J)}$ ne contient aucun poids $\tau\left(J^{\prime}\right) \in \mathcal{D}\left(\bar{\rho}^{\mathrm{ss}}\right)$ avec $J^{\prime} \nsubseteq J$.

Démonstration. En appliquant [8, Lem. 15.3] à $\tau=\tau(J), \sigma^{\prime}=\tau(J)$ et $\sigma^{\max }=\tau\left(J_{\bar{\rho}}\right)$, on déduit que le poids $\tau(J)$ apparaît sur la composante $D_{\tau\left(J \cap J_{\bar{\rho}}\right)}(\bar{\rho})$ de $D(\bar{\rho})$. L'existence de $W_{\tau(J)}$ est donc assurée par [8, Cor. 3.12], où il est noté par $I\left(\tau\left(J \cap J_{\bar{\rho}}\right), \tau(J)\right)$. La structure de $W_{\tau(J)}$ est décrite par [8, Cor. 4.11]. Plus précisément, si l'on définit $\mathcal{S}(\tau(J))$ comme dans [8, Cor. 4.11] :

$$
\mathcal{S}(\tau(J)):=\left\{i \in \mathcal{S}, \tau(J)_{i}\left(x_{i}\right)=p-2-\tau\left(J \cap J_{\bar{\rho}}\right)_{i}\left(x_{i}\right)- \pm 1, \tau\left(J \cap J_{\bar{\rho}}\right)_{i}\left(x_{i}\right) \pm 1\right\},
$$

alors les sous-quotients irréductibles de $W_{\tau(J)}$ correspondent à des sous-ensembles de $\mathcal{S}(\tau(J))$ (car une condition de compatibilité doit être imposée). Si $i \in J \backslash J_{\bar{\rho}}$, on a par définition $\tau(J)_{i}\left(x_{i}\right) \in\left\{p-3-x_{i}, x_{i}+1\right\}$ et $\tau\left(J \cap J_{\bar{\rho}}\right)_{i}\left(x_{i}\right) \in\left\{x_{i}, p-2-x_{i}\right\}$, d'où l'inclusion $J \backslash J_{\bar{\rho}} \subseteq \mathcal{S}(\tau(J))$. D'autre part, si $i \notin J \cup J_{\bar{\rho}}$, on a $\lambda_{i}\left(x_{i}\right), \tau\left(J \cap J_{\bar{\rho}}\right)_{i}\left(x_{i}\right) \in\left\{x_{i}, p-2-x_{i}\right\}$, d'où $i \notin \mathcal{S}(\tau(J))$. On en conclut donc

$$
J \backslash J_{\bar{\rho}} \subseteq \mathcal{S}(\tau(J)) \subseteq J \cup J_{\bar{\rho}}
$$

Soit maintenant $\tau\left(J^{\prime}\right) \in \mathcal{D}\left(\bar{\rho}^{\mathrm{ss}}\right)$ un poids qui apparaît comme sous-quotient dans $W_{\tau(J)}$. On doit avoir $J \cap J_{\bar{\rho}}=J^{\prime} \cap J_{\bar{\rho}}$ car $\tau(J)$ et $\tau\left(J^{\prime}\right)$ apparaissent dans la même composante de $D(\bar{\rho})$, et $\mathcal{S}\left(\tau\left(J^{\prime}\right)\right) \subseteq \mathcal{S}(\tau(J))$ d'après [8, Cor. 4.11]. Cela force que par (24) :

$$
J^{\prime} \backslash J_{\bar{\rho}} \subseteq \mathcal{S}\left(\tau\left(J^{\prime}\right)\right) \subseteq \mathcal{S}(\tau(J)) \subseteq J \cup J_{\bar{\rho}},
$$

d'où $J^{\prime} \subseteq J$.

Proposition 5.2. Il existe des sous-représentations uniques $D_{\ell}(\bar{\rho})$ de $D_{\ell}\left(\bar{\rho}^{\mathrm{sS}}\right)$ pour $0 \leq \ell \leq$ $f$, telles que $D(\bar{\rho})$ soit une suite d'extensions successives de la forme

$$
D_{0}(\bar{\rho})-D_{1}(\bar{\rho})-\cdots-D_{f}(\bar{\rho}),
$$

avec

$$
\operatorname{soc}_{\mathrm{GL}_{2}\left(\mathbb{F}_{q}\right)} D_{\ell}(\bar{\rho})=\bigoplus \tau(J)
$$

la somme étant prise pour les $J \subseteq \mathcal{S}$ tels que $|J|=\ell$. En particulier, $D_{\ell}(\bar{\rho})$ est non nul pour tout $0 \leq \ell \leq f$. 
Démonstration. On définit $D_{0}(\bar{\rho})$ comme la plus grande sous-représentation de $D(\bar{\rho})$ qui vérifie la condition $(25)$, i.e. $\operatorname{soc}_{\mathrm{GL}_{2}\left(\mathbb{F}_{q}\right)} D_{0}(\bar{\rho})=\tau(\emptyset)$, et qui ne contient pas de poids $\tau(J) \in$ $\mathcal{D}\left(\bar{\rho}^{\mathrm{ss}}\right)$ tel que $|J| \geq 1$. D'après le lemme 5.1 , le socle du quotient $D(\bar{\rho}) / D_{0}(\bar{\rho})$ contient la somme directe des poids $\tau(J) \in \mathcal{D}\left(\bar{\rho}^{\mathrm{SS}}\right)$ avec $|J|=1$ (tous ces poids apparaissent par la preuve du lemme 5.1), ce qui permet de reprendre cette construction pour définir $D_{1}(\bar{\rho})$ et conclure par récurrence. Enfin, l'inclusion de $D_{\ell}(\bar{\rho})$ dans $D_{\ell}\left(\bar{\rho}^{\text {ss }}\right)$ se déduit facilement de [8, Prop. 13.1]. En effet, par construction, $D_{\ell}(\bar{\rho})$ est de multiplicité 1 et ne contient aucun poids $\tau(J) \in \mathcal{D}\left(\bar{\rho}^{\mathrm{ss}}\right)$ tel que $|J| \neq \ell$.

Remarque 5.3. (i) La représentation $D_{\ell}(\bar{\rho})$ peut être strictement plus petite que $D_{\ell}\left(\bar{\rho}^{\mathrm{ss}}\right)$.

(ii) En général, $D_{\ell}(\bar{\rho})^{I_{1}\left(\mathcal{O}_{L}\right)}$ contient plus de caractères que ceux provenant de $D(\bar{\rho})^{I_{1}\left(\mathcal{O}_{L}\right)}$, et n'est pas forcément stable par $\left(\begin{array}{ll}0 & 1 \\ p & 0\end{array}\right)$. Ce phénomène se présente déjà dans le cas $f=2$ et $|\mathcal{D}(\bar{\rho})|=1$.

(iii) Cependant, si l'on définit $D_{\ell, 1}$ comme le sous-espace des vecteurs $v \in D_{\ell}(\bar{\rho})^{I_{1}\left(\mathcal{O}_{L}\right)}$ qui se relève en un vecteur de $D(\bar{\rho})^{I_{1}\left(\mathcal{O}_{L}\right)}$, alors $D_{\ell, 1}$ est stable par $\left(\begin{array}{ll}0 & 1 \\ p & 0\end{array}\right)$.

Décrivons maintenant le sous-ensemble des poids de $\mathcal{D}\left(\bar{\rho}^{\mathrm{ss}}\right)$ qui contribuent à l'espace $D(\bar{\rho})^{I_{1}\left(\mathcal{O}_{L}\right)}$. D'après les rappels au début du $\S 2$, ce sont exactement les poids $\tau(\lambda)$ avec $\lambda \in \mathcal{P D}^{\mathrm{SS}}\left(x_{0}, \cdots, x_{f-1}\right)$, où par définition

$\mathcal{P D}^{\mathrm{ss}}\left(x_{0}, \cdots, x_{f-1}\right):=\left\{\lambda \in \mathcal{P D}\left(x_{0}, \cdots, x_{f-1}\right), \lambda_{i}\left(x_{i}\right) \in\left\{x_{i}, x_{i}+1, p-2-x_{i}, p-3-x_{i}\right\}\right\}$.

Remarquons que c'est aussi l'ensemble des $f$-uplets $\lambda$ vérifiant les conditions (i)-(iii) de la définition de $\mathcal{D}\left(x_{0}, \cdots, x_{f-1}\right)$ (cf. $\S 2$ ) pour lesquels (iv) est remplacé par : $\lambda_{i}\left(x_{i}\right)=p-3-x_{i}$ implique $i \in J_{\bar{\rho}}$. On en déduit que

$$
\mathcal{D}\left(x_{0}, \cdots, x_{f-1}\right) \subseteq \mathcal{P D}^{\mathrm{ss}}\left(x_{0}, \cdots, x_{f-1}\right) \subseteq \mathcal{P} \mathcal{D}\left(x_{0}, \cdots, x_{f-1}\right) .
$$

Dans la suite, on fixe un diagramme de Diamond $D(\bar{\rho}, r)$ associé à $\bar{\rho}$ (cf. [8]), ce qui revient à se donner une action de $\left(\begin{array}{ll}0 & 1 \\ p & 0\end{array}\right)$ sur $D(\bar{\rho})^{I_{1}\left(\mathcal{O}_{L}\right)}$ et à prendre $r: D(\bar{\rho})^{I_{1}\left(\mathcal{O}_{L}\right)} \hookrightarrow D(\bar{\rho})$ l'inclusion naturelle. Rappelons que $p$ agit trivialement sur $D(\bar{\rho})$.

Notons encore $\delta$ l'application bijective de $\mathcal{P D}\left(x_{0}, \cdots, x_{f-1}\right)$ sur lui-même définie par $\delta(\lambda)_{i}\left(x_{i}\right)=\lambda_{i+1}\left(x_{i}\right)$. Pour $\lambda \in \mathcal{P D}^{\text {ss }}\left(x_{0}, \cdots, x_{f-1}\right)$, on a (d'après la définition (9))

$$
I(\lambda)=\left\{i \in \mathcal{S} \mid \lambda_{i}\left(x_{i}\right) \in\left\{x_{i}+1, p-3-x_{i}\right\}\right\} .
$$

Il est évident que $\mathcal{P D}^{\mathrm{ss}}\left(x_{0}, \cdots, x_{f-1}\right)$ est stable par $\delta$ et que $I(\delta(\lambda))=\delta(I(\lambda))$.

Lemme 5.4. Soit $\lambda \in \mathcal{P D}^{\mathrm{ss}}\left(x_{0}, \cdots, x_{f-1}\right)$ et $w(\lambda) \in D(\bar{\rho})^{I_{1}\left(\mathcal{O}_{L}\right)}\left(\operatorname{resp} . w(\delta(\lambda)) \in D(\bar{\rho})^{I_{1}\left(\mathcal{O}_{L}\right)}\right)$ un vecteur de base de $\tau(\lambda)^{I_{1}\left(\mathcal{O}_{L}\right)}$ (resp. de $\left.\tau(\delta(\lambda))^{I_{1}\left(\mathcal{O}_{L}\right)}\right)$.

(i) Il existe un unique entier $s(\lambda) \in\{0, \cdots, q-1\}$ tel que

$$
\sum_{t \in \mathbb{F}_{q}} \sigma_{0}(t)^{s(\lambda)}\left(\begin{array}{cc}
{[t]} & 1 \\
1 & 0
\end{array}\right)\left(\begin{array}{ll}
0 & 1 \\
p & 0
\end{array}\right) w(\lambda) \in k_{E}^{\times} w(\delta(\lambda)) .
$$

(ii) On a explicitement

$$
s(\lambda)=\sum_{\{j \mid j \in \delta(I(\lambda)), j+1 \notin \delta(I(\lambda))\}} p^{j}\left(p-2-r_{j}\right)+\sum_{\{j \mid j \notin \delta(I(\lambda)), j+1 \in \delta(I(\lambda))\}} p^{j}\left(r_{j}+1\right) .
$$


(iii) Si $J_{\bar{\rho}}=\emptyset$, on a

$$
s(\lambda)=\sum_{j \in \delta(I(\lambda))} p^{j}\left(p-2-r_{j}\right)+\sum_{j \in \delta^{2}(I(\lambda))} p^{j}\left(r_{j}+1\right)
$$

et

$$
s(\lambda)-\sum_{j \notin \delta(I(\lambda)) \cup \delta^{2}(I(\lambda))} p^{j}\left(p-1-r_{j}\right) \equiv \sum_{j=0}^{f-1} p^{j} \lambda_{j}\left(r_{j}\right) \quad \bmod \left(p^{f}-1\right) .
$$

Démonstration. (i) Rappelons (§2) que $\lambda^{[s]}$ désigne le $f$-uplet $\left(p-1-\lambda_{i}\left(x_{i}\right)\right)_{i}$ correspondant à $\left(\begin{array}{ll}0 & 1 \\ p & 0\end{array}\right) w(\lambda)$. Par la preuve de [4, Prop. 5.1], il suffit de montrer que la représentation $\left\langle\mathrm{GL}_{2}\left(\mathbb{F}_{q}\right) \cdot\left(\begin{array}{ll}0 & 1 \\ p & 0\end{array}\right) w(\lambda)\right\rangle$ admet $\tau(\delta(\lambda))$ comme sous-quotient. Dans le cas où $\bar{\rho}$ est scindée, l'énoncé se déduit de [8, Lem. 15.2], qui nous dit que le poids $\tau\left(\lambda^{[s]}\right)$ apparaît sur la composante $D_{\tau(\delta(\lambda))}(\bar{\rho})$. Le cas général se déduit alors de la proposition 5.2. En effet, en notant $\ell=\left|J_{\lambda}\right|$, le morphisme naturel $\operatorname{Ind}_{I\left(\mathcal{O}_{L}\right)}^{\mathrm{GL}_{2}\left(\mathcal{O}_{L}\right)} \chi_{\lambda}^{s} \rightarrow D_{\ell}\left(\bar{\rho}^{\mathrm{Ss}}\right)$ se factorise par l'inclusion $D_{\ell}(\bar{\rho}) \hookrightarrow D_{\ell}\left(\bar{\rho}^{\mathrm{ss}}\right)$, donc le même entier $s(\lambda)$ sert comme dans le cas scindé.

(ii) Déterminons l'entier $s(\lambda)$. On a les relations suivantes :

- si $\delta(\lambda)_{i}\left(x_{i}\right) \in\left\{x_{i}, p-2-x_{i}\right\}$, alors $\lambda_{i}\left(x_{i}\right) \in\left\{x_{i}, x_{i}+1\right\}$ et $\lambda_{i}^{[s]}\left(x_{i}\right) \in\left\{p-1-x_{i}, p-\right.$ $\left.2-x_{i}\right\}$

- si $\delta(\lambda)_{i}\left(x_{i}\right) \in\left\{x_{i}+1, p-3-x_{i}\right\}$, alors $\lambda_{i}\left(x_{i}\right) \in\left\{p-2-x_{i}, p-3-x_{i}\right\}$ et $\lambda_{i}^{[s]}\left(x_{i}\right) \in$ $\left\{x_{i}+1, x_{i}+2\right\}$.

Grâce à [8, Lem. 2.7], on en déduit que

$$
\begin{aligned}
& s(\lambda)=\sum_{\left\{j \mid \delta(\lambda)_{j}\left(x_{j}\right) \in\left\{x_{j}+1, p-2-x_{j}\right\}\right\}} p^{j}\left(p-1-\delta(\lambda)_{j}\left(r_{j}\right)\right) \\
& \quad=\sum_{\{j \mid j \in \delta(I(\lambda)), j+1 \notin \delta(I(\lambda))\}} p^{j}\left(p-2-r_{j}\right)+\sum_{\{j \mid j \notin \delta(I(\lambda)), j+1 \in \delta(I(\lambda))\}} p^{j}\left(r_{j}+1\right) .
\end{aligned}
$$

(iii) Pour la première égalité, il suffit de noter que lorsque $J_{\bar{\rho}}=\emptyset, I(\lambda)$ n'est autre que $\left\{i \in \mathcal{S} \mid \lambda_{i}\left(x_{i}\right)=x_{i}+1\right\}$ et que $i \in I(\lambda)$ implique automatiquement $i-1, i+1 \notin I(\lambda)$. Pour la deuxième, on note d'abord que $j \in \delta(I(\lambda))$ si et seulement si $\lambda_{j}\left(x_{j}\right)=p-2-x_{j}$, donc il reste à prouver

$$
\sum_{j \in \delta^{2}(I(\lambda))} p^{j}\left(r_{j}+1\right)-\sum_{j \notin \delta(I(\lambda)) \cup \delta^{2}(I(\lambda))} p^{j}\left(p-1-r_{j}\right) \equiv \sum_{j \notin \delta(I(\lambda))} p^{j} \lambda_{j}\left(r_{j}\right) \bmod \left(p^{f}-1\right),
$$

soit en notant que $\delta(I(\lambda)) \cap \delta^{2}(I(\lambda))=\emptyset$ :

$$
\sum_{j \notin \delta(I(\lambda))} p^{j} r_{j}+\sum_{j \in \delta^{2}(I(\lambda))} p^{j}-\sum_{j \notin \delta(I(\lambda)) \cup \delta^{2}(I(\lambda))} p^{j}(p-1) \equiv \sum_{j \notin \delta(I(\lambda))} p^{j} \lambda_{j}\left(r_{j}\right) \bmod \left(p^{f}-1\right) .
$$

Par définition de $\mathcal{P} \mathcal{D}^{\text {ss }}\left(x_{0}, \cdots, x_{f-1}\right)$, on peut décomposer l'ensemble $\mathcal{S} \backslash \delta(I(\lambda))$ comme réunion disjointe de sous-ensembles sous la forme suivante :

$$
\mathcal{S} \backslash \delta(I(\lambda))=\coprod_{i=1}^{m}\left\{j_{i}, j_{i}+1, \cdots, j_{i}+n_{i}\right\}
$$


avec, pour chaque $1 \leq i \leq m$ :

$j_{i}, \ldots, j_{i}+\left(n_{i}-1\right) \notin \delta(I(\lambda)) \cup \delta^{2}(I(\lambda)), \quad j_{i}+n_{i} \in \delta^{2}(I(\lambda)), \quad j_{i}-1, j_{i}+n_{i}+1 \in \delta(I(\lambda))$,

ce qui implique que

$$
\lambda_{j_{i}}\left(x_{j_{i}}\right)=x_{j_{i}}+1, \quad \lambda_{j_{i}+k}\left(x_{j_{i}+k}\right)=x_{j_{i}+k}, 1 \leq k \leq n_{i} .
$$

Par conséquent, on obtient

$$
\begin{aligned}
\sum_{j \notin \delta(I(\lambda))} p^{j} r_{j}+\sum_{j \in \delta^{2}(I(\lambda))} p^{j} & -\sum_{j \notin \delta(I(\lambda)) \cup \delta^{2}(I(\lambda))} p^{j}(p-1) \\
& \equiv \sum_{j \notin \delta(I(\lambda))} p^{j} r_{j}+\sum_{i=1}^{m} p^{j_{i}}=\sum_{j \notin \delta(I(\lambda))} p^{j} \lambda_{j}\left(r_{j}\right) \bmod \left(p^{f}-1\right),
\end{aligned}
$$

où la congruence vient du fait que l'on a identifié $f$ avec 0 dans la décomposition (26). Cela termine la preuve.

Soit $\lambda \in \mathcal{P D}^{\mathrm{ss}}\left(x_{0}, \cdots, x_{f-1}\right)$. On note $d_{\lambda}$ la longueur de l'orbite de $\delta$ contenant $\lambda$, i.e. le plus petit entier positif $d$ tel que $\delta^{d}(\lambda)=\lambda$ et $\delta^{d-1}(\lambda) \neq \lambda$. Le lemme 5.4 permet de définir un morphisme pour tout $0 \leq i \leq d_{\lambda}-1$ :

$$
\begin{aligned}
& S: \quad \tau\left(\delta^{i}(\lambda)\right)^{I_{1}\left(\mathcal{O}_{L}\right)} \rightarrow \tau\left(\delta^{i+1}(\lambda)\right)^{I_{1}\left(\mathcal{O}_{L}\right)} \\
& w\left(\delta^{i}(\lambda)\right) \quad \mapsto \sum_{t \in \mathbb{F}_{q}} \sigma_{0}(t)^{s\left(\delta^{i}(\lambda)\right)}\left(\begin{array}{cc}
p[t] \\
0 & 1
\end{array}\right) w\left(\delta^{i}(\lambda)\right)
\end{aligned}
$$

ainsi qu'un isomorphisme

$$
S^{d_{\lambda}}: \tau(\lambda)^{I_{1}\left(\mathcal{O}_{L}\right) \stackrel{\sim}{\rightarrow}} \tau(\lambda)^{I_{1}\left(\mathcal{O}_{L}\right)} .
$$

L'espace $\tau(\lambda)^{I_{1}\left(\mathcal{O}_{L}\right)}$ étant de dimension 1 sur $k_{E}, S^{d_{\lambda}}$ est donné par la multiplication par un scalaire $\nu(\lambda) \in k_{E}^{\times}$. Il est clair que $\nu(\lambda)$ ne dépend pas de $w(\lambda)$ et que l'on a $\nu(\lambda)=\nu\left(\delta^{i}(\lambda)\right)$ pour tout $0 \leq i \leq d_{\lambda}-1$.

Remarque 5.5. Le paramètre $\nu(\lambda)$ est un avatar de celui défini pour $\bar{\rho}^{\mathrm{ss}}$ dans $[4, \xi 6]$ (voir aussi [5, Question 9.5(i)]), on le nomme donc le paramètre cyclique associé à $\lambda$. Notre résultat principal ci-après dit que l'on trouve exactement la valeur privilégiée qui y est prédite dans le cas particulier $J_{\bar{\rho}}=\emptyset$.

Le résultat principal de cette section est le suivant, et la démonstration occupera le reste de cette section.

Théorème 5.6. Supposons que $J_{\bar{\rho}}=\emptyset$. Soient $\lambda \in \mathcal{P D}^{\mathrm{ss}}\left(x_{0}, \cdots, x_{f-1}\right)$ et $d_{\lambda}, \nu(\lambda)$ comme ci-dessus. Fixons un diagramme de Diamond $D(\bar{\rho}, r)$ et supposons que les valeurs de $x\left(\delta^{i}(\lambda)\right)$ pour tout $0 \leq i \leq d_{\lambda}-1$ vérifient l'égalité (23) du théorème 4.7. On a alors

$$
\nu(\lambda)=(-1)^{\frac{d_{\lambda}|I(\lambda)|}{f}} \sum_{j=0}^{f-1} r_{j} \xi^{(f-2|I(\lambda)|) \frac{d_{\lambda}}{f}},
$$

où l'on rappelle que $\xi$ est défini en (1). 
Remarque 5.7. Avec les notations de [4, §6], on voit que si $c_{i}$ est l'orbite de $\delta=\delta_{\text {réd }}$ contenant $\lambda$, l'ensemble $J_{i}(\subseteq \mathcal{S})$ correspondant à $\lambda$ est juste $\left\{j \in \mathcal{S} \mid \lambda_{j}\left(x_{j}\right)=p-2-x_{j}\right\}=$ $\delta(I(\lambda))$. Comme $J_{i} \cap \delta\left(J_{i}\right)=\emptyset$, on en déduit que :

$$
h_{i} \stackrel{\text { déf }}{=} \frac{1}{2}\left|\left(J_{i} \cup \delta\left(J_{i}\right)\right) \backslash\left(J_{i} \cap \delta\left(J_{i}\right)\right)\right|=\left|J_{i}\right|=|I(\lambda)| .
$$

Ainsi le théorème 5.6 donne exactement la valeur privilégiée de $\nu(\lambda)$ annoncée dans [4, Thm. 6.4].

On suppose dans le reste de la section que $J_{\bar{\rho}}=\emptyset$, ce qui entraîne que $\mathcal{D}(\bar{\rho})=\{\tau(\emptyset)=$ $\left.\left(r_{0}, \cdots, r_{f-1}\right)\right\}$ et que $D(\bar{\rho})$ s'injecte dans $\operatorname{Inj}_{\mathrm{GL}_{2}\left(\mathbb{F}_{q}\right)} \tau(\emptyset)$. La définition suivante est analogue à celle de $S$ issue du lemme 5.4 .

Définition 5.8. On définit un morphisme $R: D(\bar{\rho})^{I_{1}\left(\mathcal{O}_{L}\right)}=\left(\operatorname{Inj}_{\mathrm{GL}_{2}\left(\mathbb{F}_{q}\right)} \tau(\emptyset)\right)^{I_{1}\left(\mathcal{O}_{L}\right)} \rightarrow$ $\tau(\emptyset)^{I_{1}\left(\mathcal{O}_{L}\right)}$ de la façon suivante : si $w(\lambda) \in D(\bar{\rho})^{I_{1}\left(\mathcal{O}_{L}\right)}$ est un vecteur propre de $I\left(\mathcal{O}_{L}\right)$ de caractère $\chi$ correspondant à $\lambda \in \mathcal{P D}\left(x_{0}, \cdots, x_{f-1}\right)$, on pose (cf. [8, Prop. 2.7])

$$
R(w(\lambda)):=\sum_{t \in \mathbb{F}_{q}}\left(\prod_{j \in \mathcal{R}(\lambda)} \sigma_{0}(t)^{p^{j}\left(p-1-r_{j}\right)}\right)\left(\begin{array}{cc}
{[t]} & 1 \\
1 & 0
\end{array}\right) w(\lambda)
$$

où $\mathcal{R}(\lambda):=\left\{i \in \mathcal{S} \mid \lambda_{i}\left(x_{i}\right) \in\left\{x_{i}, x_{i}+1\right\}\right\}$.

L'opérateur $R$ permet de réécrire la proposition 2.6 sous la forme suivante (en notant que le vecteur $w(\lambda)$ correspond à $\left(\begin{array}{ll}0 & 1 \\ p & 0\end{array}\right) v(\lambda)$ et que $p$ agit trivialement sur $\pi$ qui contient $D(\bar{\rho}))$ :

$$
R\left(\left(\begin{array}{ll}
0 & 1 \\
p & 0
\end{array}\right) w(\lambda)\right)=x(\lambda)^{-1} R(w(\lambda)) .
$$

Notons d'ailleurs que si $\lambda \in \mathcal{P D}^{\mathrm{ss}}\left(x_{0}, \cdots, x_{f-1}\right)$, alors $\mathcal{R}(\lambda)=\mathcal{S} \backslash \delta(I(\lambda))$ et $\mathcal{R}\left(\lambda^{[s]}\right)=$ $\delta(I(\lambda))$.

Lemme 5.9. Soient $\lambda \in \mathcal{P D}^{\mathrm{ss}}\left(x_{0}, \cdots, x_{f-1}\right)$ et $w(\lambda)$ un vecteur propre de caractère $\chi$ correspondant $\grave{a} \lambda$. On a la relation suivante :

$$
(R \circ S)(w(\lambda))=x(\lambda)^{-1} y(\lambda) R(w(\lambda))
$$

où $y(\lambda)=(-1)^{Y(\lambda)}$ avec

$$
Y(\lambda)=1+\sum_{j \in \delta(I(\lambda))} p^{j}\left(p-2-r_{j}\right)+\sum_{j \in \delta^{2}(I(\lambda))} p^{j}\left(r_{j}+1\right)+e(\lambda)\left(r_{0}, \cdots, r_{f-1}\right) .
$$

Démonstration. Par (27), il suffit de montrer que $(R \circ S)(w(\lambda))=y(\lambda) R\left(\left(\begin{array}{ll}0 & 1 \\ p & 0\end{array}\right) w(\lambda)\right)$. Comme $\mathcal{R}(\lambda)=\mathcal{S} \backslash \delta(I(\lambda))$ pour $\lambda \in \mathcal{P} \mathcal{D}^{\text {ss }}\left(x_{0}, \cdots, x_{f-1}\right)$, la dernière formule s'écrit explicitement sous la forme suivante :

$$
\begin{array}{r}
\text { LHS }:=\sum_{t \in \mathbb{F}_{q}}\left(\prod_{j \notin \delta^{2}(I(\lambda))} \sigma_{0}(t)^{p^{j}\left(p-1-r_{j}\right)}\right)\left(\begin{array}{cc}
{[t]} & 1 \\
1 & 0
\end{array}\right) \sum_{\mu \in \mathbb{F}_{q}} \sigma_{0}(\mu)^{s(\lambda)}\left(\begin{array}{cc}
{[\mu]} & 1 \\
1 & 0
\end{array}\right)\left(\begin{array}{ll}
0 & 1 \\
p & 0
\end{array}\right) w(\lambda) \\
=y(\lambda) \sum_{t \in \mathbb{F}_{q}}\left(\prod_{j \in \delta(I(\lambda))} \sigma_{0}(t)^{p^{j}\left(p-1-r_{j}\right)}\right)\left(\begin{array}{cc}
{[t]} & 1 \\
1 & 0
\end{array}\right)\left(\begin{array}{ll}
0 & 1 \\
p & 0
\end{array}\right) w(\lambda) .
\end{array}
$$


Les deux termes donnant le même caractère grâce au lemme 5.4(iii), il suffit de déterminer le scalaire non nul $y(\lambda)$. En utilisant la formule

$$
\begin{aligned}
\left(\begin{array}{cc}
{[t]} & 1 \\
1 & 0
\end{array}\right)\left(\begin{array}{cc}
{[\mu]} & 1 \\
1 & 0
\end{array}\right) & =\left(\begin{array}{cc}
1 & {[t]} \\
0 & 1
\end{array}\right) \text { si } \mu=0 \\
& =\left(\begin{array}{cc}
{[t]+\left[\mu^{-1}\right]} & 1 \\
1 & 0
\end{array}\right)\left(\begin{array}{cc}
{[\mu]} & 1 \\
0 & -\left[\mu^{-1}\right]
\end{array}\right) \text { si } \mu \neq 0
\end{aligned}
$$

et en notant que

$$
\begin{aligned}
\left(\begin{array}{cc}
{[\mu]} & 1 \\
0 & -\left[\mu^{-1}\right]
\end{array}\right)\left(\begin{array}{ll}
0 & 1 \\
p & 0
\end{array}\right) w(\lambda) & =\left(\begin{array}{cc}
0 & 1 \\
p & 0
\end{array}\right)\left(\begin{array}{cc}
-\left[\mu^{-1}\right] & 0 \\
p & {[\mu]}
\end{array}\right) w(\lambda) \\
& =\left(-\sigma_{0}(\mu)\right)^{-\sum_{j=0}^{f-1} p^{j} \lambda_{j}\left(r_{j}\right)}(-1)^{e(\lambda)\left(r_{0}, \cdots, r_{f-1}\right)}\left(\begin{array}{ll}
0 & 1 \\
p & 0
\end{array}\right) w(\lambda),
\end{aligned}
$$

on voit que le terme LHS de $(28)$ est égal à $(\operatorname{car} s(\lambda) \neq 0)$ :

$$
\begin{aligned}
& (-1)^{z} \sum_{t \in \mathbb{F}_{q}, \mu \in \mathbb{F}_{q}^{\times}} \sigma_{0}(t)^{j \notin \delta^{2}(I(\lambda))} p^{j\left(p-1-r_{j}\right)} \sigma_{0}(\mu)^{s(\lambda)-\sum_{j=0}^{f-1} p^{j} \lambda_{j}\left(r_{j}\right)}\left(\begin{array}{cc}
{[t]+\left[\mu^{-1}\right]} & 1 \\
1 & 0
\end{array}\right)\left(\begin{array}{ll}
0 & 1 \\
p & 0
\end{array}\right) w(\lambda) \\
= & (-1)^{z} \sum_{t \in \mathbb{F}_{q}, \mu \in \mathbb{F}_{q}^{\times}} \sigma_{0}(t-\mu)^{j \notin \delta^{2}(I(\lambda))} p^{j\left(p-1-r_{j}\right)} \sigma_{0}(\mu)^{-s(\lambda)+\sum_{j=0}^{f-1} p^{j} \lambda_{j}\left(r_{j}\right)}\left(\begin{array}{cc}
t] & 1 \\
1 & 0
\end{array}\right)\left(\begin{array}{ll}
0 & 1 \\
p & 0
\end{array}\right) w(\lambda)
\end{aligned}
$$

où l'on a noté $z:=e(\lambda)\left(r_{0}, \cdots, r_{f-1}\right)-\sum_{j=0}^{f-1} p^{j} \lambda_{j}\left(r_{j}\right)$. Comme $\delta(I(\lambda)) \cap \delta^{2}(I(\lambda))=\emptyset$, le lemme 5.4(iii) implique que

$$
\sum_{j \notin \delta^{2}(I(\lambda))} p^{j}\left(p-1-r_{j}\right)-s(\lambda)+\sum_{j=0}^{f-1} p^{j} \lambda_{j}\left(r_{j}\right) \equiv \sum_{j \in \delta(I(\lambda))} p^{j}\left(p-1-r_{j}\right) \bmod \left(p^{f}-1\right),
$$

d'où $\sum_{\mu \in \mathbb{F}_{q}^{\times}} \sigma_{0}(-\mu)^{\sum_{j \notin \delta^{2}(I(\lambda))} p^{j}\left(p-1-r_{j}\right)} \sigma_{0}(\mu)^{-s(\lambda)+\sum_{j=0}^{f-1} p^{j} \lambda_{j}\left(r_{j}\right)}=0$ et

$$
\begin{aligned}
& \text { LHS }=(-1)^{z} \sum_{t \in \mathbb{F}_{q}} \sigma_{0}(t)^{\sum_{j \in \delta(I(\lambda))} p^{j}\left(p-1-r_{j}\right)} . \\
& {\left[\sum_{\mu \in \mathbb{F}_{q}} \sigma_{0}(1-\mu)^{\sum_{j \notin \delta^{2}(I(\lambda))} p^{j\left(p-1-r_{j}\right)}} \sigma_{0}(\mu)^{-s(\lambda)+\sum_{j=0}^{f-1} p^{j} \lambda_{j}\left(r_{j}\right)}\right]\left(\begin{array}{cc}
{[t]} & 1 \\
1 & 0
\end{array}\right)\left(\begin{array}{ll}
0 & 1 \\
p & 0
\end{array}\right) w(\lambda) .}
\end{aligned}
$$

Puis, en appliquant (la version modulo $p$ ) de [6, Thm. 2.5.1] avec

$$
a=\sum_{j \notin \delta(I(\lambda)) \cup \delta^{2}(I(\lambda))} p^{j} r_{j}+\sum_{j \in \delta(I(\lambda)) \cup \delta^{2}(I(\lambda))} p^{j}(p-1), \quad b=\sum_{j \notin \delta^{2}(I(\lambda))} p^{j}\left(p-1-r_{j}\right),
$$

on trouve

$$
\sum_{\mu \in \mathbb{F}_{q}} \sigma_{0}(1-\mu)^{\sum_{j \notin \delta^{2}(I(\lambda))} p^{j}\left(p-1-r_{j}\right)} \sigma_{0}(\mu)^{-s(\lambda)+\sum_{j=0}^{f-1} p^{j} \lambda_{j}\left(r_{j}\right)}=(-1)^{1-\sum_{j \notin \delta(I(\lambda)) \cup \delta^{2}(I(\lambda))} p^{j}\left(p-1-r_{j}\right)}
$$

ce qui donne le résultat grâce au lemme 5.4(iii). 
Démonstration du théorème 5.6. Remarquons d'abord que $\nu(\lambda)$ est aussi l'unique scalaire solution de l'équation suivante:

$$
\left(R \circ S^{d_{\lambda}}\right)(w(\lambda))=\nu(\lambda) \cdot R(w(\lambda)) .
$$

En appliquant le lemme 5.9 successivement, on obtient

$$
\nu(\lambda)=\prod_{i=0}^{d_{\lambda}-1} x\left(\delta^{i}(\lambda)\right)^{-1} y\left(\delta^{i}(\lambda)\right)
$$

Le théorème 4.7 appliqué au cas $J_{\bar{\rho}}=\emptyset$ implique que

$$
x\left(\delta^{i}(\lambda)\right)=(-1) \frac{\sqrt[f]{\xi}^{\left|I\left(\delta^{i}(\lambda)\right)\right|}}{\sqrt[f]{\xi}\left|\mathcal{S} \backslash I\left(\delta^{i}(\lambda)\right)\right|} \frac{\prod_{j \in I\left(\delta^{i}(\lambda)\right)}\left(r_{j}+1\right) \mu_{f-j}}{\prod_{j \in I\left(\delta^{i+1}(\lambda)\right)}\left(r_{j}+1\right) \mu_{f-j}},
$$

d'où l'on obtient, en notant que $\left|I\left(\delta^{i}(\lambda)\right)\right|=|I(\lambda)|$, que

$$
\prod_{i=0}^{d_{\lambda}-1} x\left(\delta^{i}(\lambda)\right)=(-1)^{d_{\lambda}} \frac{1}{\sqrt[f]{\xi} d^{d_{\lambda}(f-2|I(\lambda)|)}}=(-1)^{d_{\lambda}} \xi^{-(f-2|I(\lambda)|) \frac{d_{\lambda}}{f}} .
$$

D'autre part, le lemme 5.9 implique $\prod_{i=0}^{d_{\lambda}-1} y\left(\delta^{i}(\lambda)\right)=(-1)^{Y}$ avec

$$
\begin{aligned}
Y & =\sum_{i=0}^{d_{\lambda}-1} Y\left(\delta^{i}(\lambda)\right) \\
& =\sum_{i=0}^{d_{\lambda}-1}\left[1+\sum_{j \in \delta^{i+1}(I(\lambda))} p^{j}\left(p-2-r_{j}\right)+\sum_{j \in \delta^{i+2}(I(\lambda))} p^{j}\left(r_{j}+1\right)+e\left(\delta^{i}(\lambda)\right)\left(r_{0}, \cdots, r_{f-1}\right)\right] \\
& =d_{\lambda}+\sum_{i=0}^{d_{\lambda}-1} p^{j}(p-1)+\sum_{i=0}^{d_{\lambda}-1} e\left(\delta^{i}(\lambda)\right)\left(r_{0}, \cdots, r_{f-1}\right) \\
& \equiv d_{\lambda}+\sum_{i=0}^{d_{\lambda}-1} e\left(\delta^{i}(\lambda)\right)\left(r_{0}, \cdots, r_{f-1}\right) \bmod 2 .
\end{aligned}
$$

On vérifie à partir de la définition $[8, \S 2]$ que

$$
e(\lambda)\left(r_{0}, \cdots, r_{f-1}\right)=\sum_{j \in I(\lambda)} p^{j}(-1)+\sum_{j \in \delta(I(\lambda))} p^{j}\left(r_{j}+1\right)
$$

d'où

$$
\sum_{i=0}^{d_{\lambda}-1} e\left(\delta^{i}(\lambda)\right)\left(r_{0}, \cdots, r_{f-1}\right)=\sum_{i=0}^{d_{\lambda}-1} \sum_{j \in I\left(\delta^{i}(\lambda)\right)} p^{j} r_{j} \equiv \frac{d_{\lambda}|I(\lambda)|}{f}\left(\sum_{j=0}^{f-1} r_{j}\right) \bmod 2 .
$$

Le résultat se déduit alors de (29).

Remarque 5.10. Le théorème 4.7 ne permet de calculer $\nu(\lambda)$ que lorsque $J_{\bar{\rho}}=\emptyset$. Pour pouvoir l'appliquer, il faut en effet que tous les poids $\tau\left(\delta^{i}(\lambda)\right)$, avec $\lambda \in \mathcal{P D}^{\mathrm{ss}}\left(x_{0}, \cdots, x_{f-1}\right)$ et $0 \leq i \leq d_{\lambda}-1$, apparaissent dans une même composante $D_{\tau}(\bar{\rho})$ pour $\tau \in \mathcal{D}(\bar{\rho})$. Cela nécessite, d'après la proposition 2.1, que pour tout $0 \leq i \leq d_{\lambda}-1$, on ait

$$
J_{\tau}=\left\{j \mid\left(\delta^{i}(\lambda)\right)_{j}\left(x_{j}\right)=p-3-x_{j}\right\},
$$


et donne donc deux cas : soit $J_{\tau}=\emptyset$ si $\lambda_{j}\left(x_{j}\right) \neq p-3-x_{j}$ pour tout $j$, soit $J_{\tau}=\mathcal{S}$ sinon. Ce dernier cas n'est cependant pas intéressant car il correspond à $\lambda=\left(p-3-x_{0}, \cdots, p-\right.$ $\left.3-x_{f-1}\right)$ et $d_{\lambda}=1$.

Rappelons que $D_{\ell}(\bar{\rho})$ est le sous-quotient de $D(\bar{\rho})$ défini dans la proposition 5.2. Notons $D_{\ell}:=\left(D_{\ell, 0}, D_{\ell, 1}, r_{\ell}\right)$ le diagramme induit, c'est-à-dire, on prend $D_{\ell, 0}:=D_{\ell}(\bar{\rho}), D_{\ell, 1}$ le sous-espace de $\left(D_{\ell, 0}\right)^{I_{1}\left(\mathcal{O}_{L}\right)}$ défini dans la remarque 5.3(iii), et le morphisme $r_{\ell}: D_{\ell, 1} \rightarrow$ $D_{\ell, 0}$ est l'inclusion naturelle. Si $D_{\ell, 1} \neq 0$, alors il est stable par l'opérateur $S$ défini plus haut, et si $\lambda \in \mathcal{P} \mathcal{D}^{\mathrm{ss}}\left(x_{0}, \cdots, x_{f-1}\right)$ fournit un caractère apparaissant sur $D_{\ell, 1}$ (en fait sur $\left.\left(\operatorname{soc}_{\mathrm{GL}}\left(\mathcal{O}_{F}\right) D_{\ell, 0}\right)^{I_{1}\left(\mathcal{O}_{L}\right)}\right)$, alors le scalaire $\nu(\lambda) \in k_{E}^{\times}$associé à $S^{d_{\lambda}}$ est bien défini.

On peut reformuler le théorème 5.6 sous la forme suivante.

Théorème 5.11. Supposons que $J_{\bar{\rho}}=\emptyset$ et que pour tout $\lambda \in \mathcal{P D}^{\mathrm{ss}}\left(x_{0}, \cdots, x_{f-1}\right)$, les valeurs de $x(\lambda)$ vérifient l'égalité (23) du théorème 4.7. Alors le diagramme $D(\bar{\rho}, r)$ est suite d'extensions successives de la forme (dans la catégories des diagrammes, voir [8, §9])

$$
D_{0}-D_{1}-\cdots-D_{f}
$$

De plus, le paramètre cyclique $\nu(\lambda)$ a la valeur prédite dans [4, Thm. 6.4(i)].

Remarque 5.12. Supposons que $J_{\bar{\rho}}=\emptyset$. Bien que $D_{\ell, 0}$ est toujours non nul, on a $D_{\ell, 1}=0$ pour $\ell \geq\lfloor f / 2\rfloor+1$. En effet, sinon, il existe $\lambda \in \mathcal{P D}^{\mathrm{ss}}\left(x_{0}, \cdots, x_{f-1}\right)$ tel que $\left|J_{\lambda}\right| \geq\lfloor f / 2\rfloor+1$, mais ce n'est pas possible, car $i \in J_{\lambda}$ entraîne $i+1, i-1 \notin J_{\lambda}$, ainsi $\left|J_{\lambda}\right| \leq\lfloor f / 2\rfloor$.

\section{$6 \quad$ Nombre de paramètres}

On détermine le nombre de paramètres permettant de classifier les diagrammes de Diamond associés à $\bar{\rho}$.

On commence par choisir une base de $D(\bar{\rho})^{I_{1}\left(\mathcal{O}_{L}\right)}$ de la façon relativement naturelle (notons que l'on a pas a priori de choix canonique). Comme $D(\bar{\rho})$ est somme directe $\oplus_{J \subseteq J_{\bar{\rho}}} D_{\tau(J)}(\bar{\rho})$ avec chaque $D_{\tau(J)}(\bar{\rho})$ indécomposable (en tant que $\mathrm{GL}_{2}\left(\mathcal{O}_{L}\right)$-représentation), fixer une base, disons $w(J)$, de $\tau(J)^{I_{1}\left(\mathcal{O}_{L}\right)}$ va fixer la composante $D_{\tau(J)}(\bar{\rho})$. Plus précisément, on a $\operatorname{soc}_{\mathrm{GL}_{2}\left(\mathbb{F}_{q}\right)} D_{\tau(J)}(\bar{\rho})=\tau(J)$, d'où une injection $D_{\tau(J)}(\bar{\rho}) \hookrightarrow \operatorname{Inj}_{\mathrm{GL}_{2}\left(\mathbb{F}_{q}\right)} \tau(J)$, et la définition 5.8 (avec un changement de variables) donne un morphisme

$$
R_{J}: D_{\tau(J)}(\bar{\rho})^{I_{1}\left(\mathcal{O}_{L}\right)} \rightarrow \tau(J)^{I_{1}\left(\mathcal{O}_{L}\right)}
$$

Si $\chi$ est un caractère apparaissant sur $D_{\tau(J)}(\bar{\rho})^{I_{1}\left(\mathcal{O}_{L}\right)}$ correspondant à $\lambda \in \mathcal{P D}\left(x_{0}, \cdots, x_{f-1}\right)$, on note $w(\lambda)$ l'unique vecteur tel que

$$
R_{J}(w(\lambda))=w(J) .
$$

On obtient ainsi une base $\{w(\lambda)\}_{\lambda}$ de $D(\bar{\rho})^{I_{1}\left(\mathcal{O}_{L}\right)}$ dépendant du choix de vecteurs $w(J)$ avec $J \subseteq J_{\bar{\rho}}$.

Regardons maintenant la structure du diagramme $D(\bar{\rho}, r)$, où $r: D(\bar{\rho})^{I_{1}\left(\mathcal{O}_{L}\right)} \hookrightarrow D(\bar{\rho})$ est une injection $I\left(\mathcal{O}_{L}\right) L^{\times}$-équivariante. Soit $\left\{\chi, \chi^{s}\right\}$ une paire de caractères apparaissant sur $D(\bar{\rho})^{I_{1}\left(\mathcal{O}_{L}\right)}$ et correspondant à $\left\{\lambda, \lambda^{[s]}\right\}$ avec $\lambda \in \mathcal{P D}\left(x_{0}, \cdots, x_{f-1}\right)$. On définit $x(\lambda) \in k_{E}^{\times}$ comme l'unique scalaire déterminé par l'équation

$$
\left(\begin{array}{ll}
0 & 1 \\
p & 0
\end{array}\right) w\left(\lambda^{[s]}\right)=x(\lambda) w(\lambda) .
$$


Comme $p$ agit trivialement sur $D(\bar{\rho})$, on a $x(\lambda) x\left(\lambda^{[s]}\right)=1$. Tautologiquement, avec une base de $D(\bar{\rho})^{I_{1}\left(\mathcal{O}_{L}\right)}$ fixée, se donner un morphisme $r$ est équivalent à se donner les scalaires $\{x(\lambda)\}_{\lambda \in \mathcal{P D}\left(x_{0}, \cdots, x_{f-1}\right)}$.

Remarque 6.1. Lorsque $\left\{\chi, \chi^{s}\right\}$ apparaissent tous les deux sur $D_{\tau(J)}(\bar{\rho})^{I_{1}\left(\mathcal{O}_{L}\right)}$ pour un $J \subseteq J_{\bar{\rho}}$, le scalaire $x(\lambda)$ n'est autre que celui de la proposition 2.6 (avec $\lambda$ correspondant $\grave{a}$ $\chi)$. De plus, $x(\lambda)$ ne dépend que de $D(\bar{\rho}, r)$.

Proposition 6.2. Supposons que $\bar{\rho}$ est non scindée avec $d=\left|J_{\bar{\rho}}\right|$.

(i) Fixons $D(\bar{\rho}, r)$ un diagramme de Diamond associé à $\bar{\rho}$. On peut toujours choisir une base de $D(\bar{\rho})^{I_{1}\left(\mathcal{O}_{L}\right)}$ telle que $x(\lambda)=x\left(\lambda^{[s]}\right)=1$ pour les $\lambda$ qui correspondent aux poids $\tau(J)$ vérifiant $\emptyset \neq J \subseteq J_{\bar{\rho}}$, et les autres $x(\lambda)$ sont alors uniquement déterminés par (31).

(ii) Les diagrammes $D(\bar{\rho}, r)$ sont paramétrés (à isomorphisme près) par les paramètres $\{x(\lambda)\}_{\lambda}$, à valeurs dans $k_{E}^{\times}$, vérifiant $x(\lambda) x\left(\lambda^{[s]}\right)=1$ pour tout $\lambda$ et $x(\lambda)=1$ pour les $\lambda$ correspondant aux poids $\tau(J)$ vérifiant $\emptyset \neq J \subseteq J_{\bar{\rho}}$. Le nombre de paramètres libres parmi les $x(\lambda)$ est $2^{f-1-d} 3^{d}-\left(2^{d}-1\right)$.

Démonstration. Fixons une base $w(\emptyset)$ de $\tau(\emptyset)^{I_{1}\left(\mathcal{O}_{L}\right)}$. Si $J \subseteq J_{\bar{\rho}}$ est un sous-ensemble de cardinal $\ell \geq 1$, nous allons définir une base $w(J)$ de $\tau(J)^{I_{1}\left(\mathcal{O}_{L}\right)}$ à partir de $w(\emptyset)$. Soit $1 \leq m \leq f$ l'unique entier donné par le lemme 6.3 ci-dessous. Alors $\tau\left(\delta^{i}(J)\right) \in \mathcal{D}(\bar{\rho})$ si $1 \leq i \leq m-1$ et $\tau\left(\delta^{m}(J)\right) \notin \mathcal{D}(\bar{\rho})$. Notons $w(J)$ l'unique vecteur de base de $\tau(J)^{I_{1}\left(\mathcal{O}_{L}\right)}$ tel que

$$
\left(R \circ\left(\begin{array}{ll}
0 & 1 \\
p & 0
\end{array}\right)\right)^{m}(w(J))=w\left(J_{\bar{\rho}} \cap \delta^{m}(J)\right),
$$

où $R:=\oplus_{J \subseteq J_{\bar{\rho}}} R_{J}$ désigne le morphisme induit $D(\bar{\rho})^{I_{1}\left(\mathcal{O}_{L}\right)} \rightarrow \oplus_{J \subseteq J_{\bar{\rho}}} \tau(J)$. Ce vecteur est bien défini grâce au lemme 6.3(ii). Notons que le cardinal de $J_{\bar{\rho}} \cap \delta^{m}(J)$ est strictement inférieur à $\ell$.

On obtient ainsi, par récurrence sur $\ell$, une base $w(J)$ de $\tau(J)^{I_{1}\left(\mathcal{O}_{L}\right)}$ pour tout $J \subseteq J_{\bar{\rho}}$ à partir de $w(\emptyset)$, ainsi qu'une base $\{\tau(\lambda)\}_{\lambda}$ de $D(\bar{\rho})^{I_{1}\left(\mathcal{O}_{L}\right)}$ comme construite au début de cette section. Par définition, on trouve que $x(\lambda)=1$ pour tout $\lambda$ correpondant à un poids $\tau(J) \in \mathcal{D}(\bar{\rho})$ avec $J \neq \emptyset$. De plus, il n'y a plus de liberté pour les autres paramètres $x(\lambda)$ sauf que $x(\lambda) x\left(\lambda^{[s]}\right)=1$. Comme $\operatorname{dim} D(\bar{\rho})^{I_{1}\left(\mathcal{O}_{L}\right)}=2^{f-d} 3^{d}$ et $|\mathcal{D}(\bar{\rho})|=2^{d}$, le résultat s'en déduit en utilisant le lemme 6.3(iii).

Lemme 6.3. Supposons que $\bar{\rho}$ est non scindée et que $\left|J_{\bar{\rho}}\right|=d \geq 1$. Soient $1 \leq i \leq d$ et $J \subseteq J_{\bar{\rho}}$ un sous-ensemble de cardinal $i$.

(i) Il existe un unique entier $1 \leq m \leq f$ tel que $\delta^{k}(J) \subseteq J_{\bar{\rho}}$ pour $1 \leq k \leq m-1$ et $\delta^{m}(J) \nsubseteq J_{\bar{\rho}}$.

(ii) Si $0 \leq i \leq m-1$, notons $\chi_{i}$ le caractère correspondant à $\tau\left(\delta^{i}(J)\right)$. Alors $\chi_{i}^{s}$ apparaît sur $D_{\tau\left(J_{\bar{\rho}} \cap \delta^{i+1}(J)\right)}(\bar{\rho})$.

(iii) Si $\tau \in \mathcal{D}(\bar{\rho})$, alors $\tau^{[s]} \notin \mathcal{D}(\bar{\rho})$.

Démonstration. L'hypothèse entraîne que $\cup_{m=1}^{f} \delta^{m}(J)=\mathcal{S}$ d'où l'existence de $m$ comme dans (i) puisque $J_{\bar{\rho}} \subsetneq \mathcal{S}$. Le (ii) est une conséquence de [8, Lem. 15.2,15.3]. Pour prouver (iii), rappelons que $\mathcal{D}(\bar{\rho})$ s'identifie à l'ensemble $\mathcal{D}\left(x_{0}, \cdots, x_{f-1}\right)$ défini au $\S 2$. Si $\tau$ et $\tau^{[s]}$ appartiennent tous les deux à $\mathcal{D}(\bar{\rho})$, on aurait $\tau_{i}\left(x_{i}\right) \in\left\{x_{i}+1, p-2-x_{i}\right\}$ pour tout $i \in \mathcal{S}$, d'où $J_{\tau}=\mathcal{S} \backslash J_{\tau^{[s]}}$. Cela est impossible puisque $J_{\tau}, J_{\tau^{[s]}} \subseteq J_{\bar{\rho}} \subsetneq \mathcal{S}$.

Traitons maintenant le cas où $\bar{\rho}$ est semi-simple, qui contient notamment le cas irréductible. 
Proposition 6.4. Supposons que $\bar{\rho}: \operatorname{Gal}\left(\overline{\mathbb{Q}}_{p} / L\right) \rightarrow \mathrm{GL}_{2}\left(k_{E}\right)$ soit une représentation continue semi-simple et soit $D(\bar{\rho}, r)$ un diagramme de Diamond associé à $\bar{\rho}$ dans [8]. Alors il y a s paramètres libres à valeurs dans $k_{E}^{\times}$permettant de déterminer la classe d'isomorphe de $D(\bar{\rho}, r)$, avec

$$
s= \begin{cases}\frac{3^{f}+1}{2}-2^{f}+(f+1) & \text { si } \bar{\rho} \text { est réductible scindée } \\ \frac{3^{f^{2}-1}}{2}-\left(2^{f}-1\right) & \text { si } \bar{\rho} \text { est irréductible. }\end{cases}
$$

Démonstration. Supposons que $\bar{\rho}$ est réductible scindée. Par [8, Thm. 15.4], $D(\bar{\rho})$ se décompose en somme directe $D(\bar{\rho})=\oplus_{\ell=0}^{f} D_{\ell}(\bar{\rho})$ avec, pour tout $0 \leq \ell \leq f$,

$$
\operatorname{soc}_{\mathrm{GL}_{2}\left(\mathbb{F}_{q}\right)} D_{\ell}(\bar{\rho})=\bigoplus_{J \subseteq \mathcal{S},|J|=\ell} \tau(J) .
$$

En outre, le diagramme $D(\bar{\rho}, r)$ se décompose en somme directe de sous-diagrammes indécomposables

$$
D(\bar{\rho}, r)=\bigoplus_{\ell=0}^{f} D_{\ell}\left(\bar{\rho}, r_{\ell}\right) .
$$

L'indécomposabilité des $D_{\ell}\left(\bar{\rho}, r_{\ell}\right)$ assure, par le même argument que celui de la proposition 6.2 , que le nombre de paramètres libres pour classifier $D_{\ell}\left(\bar{\rho}, r_{\ell}\right)$ est égal à :

$$
\frac{1}{2} \operatorname{dim} D_{\ell}(\bar{\rho})^{I_{1}\left(\mathcal{O}_{L}\right)}-(|\{J:|J|=\ell\}|-1) .
$$

En les sommant on obtient le nombre de paramètres libres, qui est égal à (d'après [8, Prop.14.7])

$$
\frac{1}{2}\left(3^{f}+1\right)-2^{f}+(f+1) .
$$

Le cas irréductible se démontre de manière analogue.

Remarque 6.5. Il n'y a pas de choix canonique pour les paramètres de la proposition 6.4, seul leur nombre est bien déterminé. Notons que ceux de la proposition 6.2, cas réductible non scindé, sont définis de manière artificielle. Dans [4], Breuil en a trouvé certains qui sont canoniquement définis, à savoir les "paramètres cycliques". On peut aussi voir [3, Ex.5.8] pour un exemple où $f=2$ et $\bar{\rho}$ est irréductible.

Donnons l'exemple du cas $f=3$ pour illustrer les paramètres dont nous avons discuté ci-dessus.

Exemple 6.6. Supposons $f=3$. Selon le cardinal de $J_{\bar{\rho}}$, on a les 4 cas suivants.

(i) Le cas où $J_{\bar{\rho}}=\emptyset$, c'est le cas traité dans [6]. Tous les paramètres sont déterminés.

(ii) Le cas où $\left|J_{\bar{\rho}}\right|=1$. Sans perte de généralité, on peut prendre $J_{\bar{\rho}}=\{0\}$ de telle sorte que $\mathcal{D}(\bar{\rho})=\{\tau(\emptyset), \tau(\{0\})\}$. D'après le corollaire 2.5 et la proposition 6.2, il y a 5 paramètres libres pour déterminer le diagramme $D(\bar{\rho}, r)$, et nous en avons calculé 4 , à savoir les paires $\left\{\lambda, \lambda^{[s]}\right\}$ avec

$$
\begin{gathered}
\lambda=\left(x_{0}, x_{1}, x_{2}\right), \quad \lambda=\left(x_{0}, p-2-x_{1}, x_{2}+1\right), \\
\lambda=\left(x_{0}+2, p-2-x_{1}, x_{2}+1\right), \quad \lambda=\left(x_{0}+2, x_{1}, x_{2}\right) .
\end{gathered}
$$


(iii) Le cas où $\left|J_{\bar{\rho}}\right|=2$. Sans perte de généralité, on peut prendre $J_{\bar{\rho}}=\{0,1\}$. D'après le corollaire 2.5 et la proposition 6.2, il y a 6 paramètres à déterminer et nous en avons calculé 4 .

(iv) Le cas où $\left|J_{\bar{\rho}}\right|=3$, i.e. $\bar{\rho}$ est scindée. D'après le corollaire 2.5 et la proposition 6.4 , il y a 10 paramètres à déterminer et nous en avons calculé 8 . Notons qu'il reste exactement les 2 paramètres cycliques à déterminer, voir [4] et aussi $\$ 5$.

\section{Références}

[1] L. Barthel \& R. Livné, Irreducible modular representations of $\mathrm{GL}_{2}$ of a local field, Duke Math. J. 75 (1994), 261-292.

[2] C. Breuil, Sur quelques représentations modulaires et p-adiques de $\mathrm{GL}_{2}\left(\mathbb{Q}_{p}\right) I$, Compositio Math. 138 (2003), 165-188.

[3] C. Breuil, Representations of Galois and of $\mathrm{GL}_{2}$ in characteristic $p$, cours à l'université Columbia, 2007.

[4] C. Breuil, Diagrammes de Diamond et $(\varphi, \Gamma)$-modules, Israel J. Math. 182 (2011), 349-382.

[5] C. Breuil, Sur un problème de compatibilité local-global modulo p pour $\mathrm{GL}_{2}$ (avec un appendice par L. Dembélé), J. Reine Angew. Math. 692 (2014), 1-76.

[6] C. Breuil \& F. Diamond, Formes modulaires de Hilbert modulo $p$ et valeurs d'extensions galoisiennes, Ann. Scient. E.N.S. 47 (2014), 905-974.

[7] C. Breuil \& A. Mézard, Multiplicités modulaires raffinées, Bull. Soc. Math. de France 142 (2014), 127-175.

[8] C. Breuil \& V. Paškūnas, Towards a modulo p Langlands correspondence for $\mathrm{GL}_{2}$, Memoirs of Amer. Math. Soc. 216, 2012.

[9] K. Buzzard, F. Diamond \& F. Jarvis, On Serre's conjecture for mod $\ell$ Galois representations over totally real fields, Duke Math. J. 55 (2010), 105-161.

[10] S. Chang \& F. Diamond, Extensions of rank one $(\varphi, \Gamma)$-modules and crystalline representations, Compositio Math. 147 (2011), 375-427.

[11] P. Colmez, Représentations de $\mathrm{GL}_{2}\left(\mathbb{Q}_{p}\right)$ et $(\varphi, \Gamma)$-modules, Astérisque 330 (2010), 281-509.

[12] J.-M. Fontaine \& G. Laffaille, Construction de représentations p-adiques, Ann. Scient. E.N.S. 15 (1982), 547-608.

[13] Y. Hu, Sur quelques représentations supersingulières de $\mathrm{GL}_{2}\left(\mathbb{Q}_{p^{f}}\right)$, J. Algebra, 324 (2010), 1577-1615.

[14] D. Savitt, On a conjecture of Conrad, Diamond, and Taylor, Duke Math. J. 128 (2005), 141-197.

[15] D. Savitt, Breuil modules for Raynaud schemes, J. Number Theory 128 (2008), 2939-2950.

[16] B. Schraen, Sur la présentation des représentations supersingulières de $\mathrm{GL}_{2}(F)$, J. Reine Angew. Math. 704 (2015), 187-208.

[17] M.-F. Vignéras, Le foncteur de Colmez pour GL $(2, F)$, Arithmetic geometry and automorphic forms, ALM 19, 531-557 (2011). 
IRMAR - UMR CNRS 6625

Bâtiment 22, Campus Beaulieu, 35042 Rennes cedex, France

Adresse e-mail : yongquan.hu@univ-rennes1.fr 